\title{
Appropriate Realisation of MIMO Gain-Scheduled Controllers
}

\author{
D.J.Leith \\ W.E. Leithead \\ Department of Electronic \& Electrical Engineering, \\ University of Strathclyde, \\ GLASGOW G1 1QE, U.K.
}

\begin{abstract}
The dynamic characteristics of a controller designed by the gain-scheduling approach can be strongly dependent on the realisation adopted; that is, the manner in which the local linear controller designs are combined to obtain a nonlocal controller. The purpose of the present paper is to investigate the choice of appropriate realisations for general MIMO gain-scheduled controllers. An extended local linear equivalence condition for MIMO gain-scheduled nonlinear controllers is proposed which minimises the controller nonlinearity. It is shown that, with few exceptions, it is possible to realise all gain-scheduled controllers as nonlinear controllers satisfying the extended local linear equivalence condition and requiring the controller to do so is not at all restrictive.
\end{abstract}

\section{Introduction}

Gain-scheduling control has a long pedigree and, having originated in flight control, is now widely employed in a variety of applications where high performance has to be achieved over a broad operating envelope. Traditionally, a gain-scheduled controller is adjusted with reference to a slowly-varying externally measured quantity which, in some sense, captures the nonlinear behaviour of the plant (Astrom \& Wittenmark 1989). However, the term 'gain-scheduled' is also widely applied to encompass a broad range of controllers including those where the scheduling variable varies rapidly (Shamma \& Athans 1992), where an internal state of the controller is employed to implicitly schedule the controller (Rugh 1991) and where the plant is essentially fixed but the control objectives vary (Leith \& Leithead 1994, 1996).

In these latter applications, the controllers may be strongly nonlinear and their dynamic characteristics may, in general, bear little relation to those of slowly-varying exogenously gain-scheduled controllers. Nevertheless, the controllers are linked by the design approach employed whereby a nonlinear controller is constructed by continuously interpolating, in some manner, between the members of a family of linear controllers. Each linear controller is, typically, associated with a specific equilibrium operating point of the plant and is designed to ensure that, locally to the equilibrium operating point, the performance requirements are met. (The existence of a set of equilibrium operating points which encompass the envelope of plant operation is central to most gain-scheduling arrangements and it is insufficient to restrict consideration to a single, isolated, equilibrium operating point). By employing a series expansion linearisation which, locally to the equilibrium operating point, has similar dynamics to the plant, linear techniques may be used to resolve this local design task. Continuity is, therefore, maintained with established linear design techniques for which a considerable body of experience has been accumulated.

It is known that the dynamic characteristics of a controller designed by the gain-scheduling approach can be strongly dependent on the realisation adopted; that is, the manner in which the local linear controllers are combined to obtain a non-local controller (see, for example, Leithead et al. 1991a, Leith \& Leithead 1996). Indeed, the performance improvement, in comparison to linear control, achieved by employing a gain-scheduled controller can be entirely lost when an inappropriate controller realisation is adopted (Leith \& Leithead 1996). However, analytic support for the choice of an appropriate controller realisation is rather poorly developed.

Lawrence \& Rugh (1995) propose that the controller realisation should be restricted to ensure that, at each equilibrium operating point, the dynamics of the controller series expansion linearisation matches the corresponding member of the family of linear controllers. (A similar approach is also advocated, in a more restricted context, by Kaminer et al. 1995). However, their results are of little utility (Leith \& Leithead 1996, 1997a). Leith \& Leithead (1996) propose, instead, that the controller realisation should satisfy a so-called extended local linear equivalence condition and derive generic realisations which satisfy this condition for a broad class of SISO controllers. Employing a realistic example from wind turbine regulation, it is demonstrated that realisations which satisfy the extended local linear equivalence condition provide a substantial improvement in performance in comparison with realisations which do not satisfy this condition (including those which satisfy the local linear equivalence condition of Lawrence \& Rugh 1995).

The objective of the present paper is to further develop the approach of Leith \& Leithead (1996) and, in particular, extend it to encompass the choice of an appropriate realisation for general multi-input multi-output gain-scheduled 
controllers. The paper is organised as follows. In section 2, some preliminary details are presented and, in section 3 , criteria for the selection of appropriate realisations are discussed. In section 4, various practical issues are addressed and realisations, satisfying the extended local linear equivalence criterion derived in section 3 , are investigated. The conclusions are summarised in section 5.

\section{Gain-scheduling design}

The traditional exogenously gain-scheduled controller, which is adjusted with reference to an externally measured variable, $\rho(t)$, has the form

$$
\mathbf{x}=\mathbf{A}(\rho(\mathrm{t})) \mathbf{x}+\mathbf{B}(\rho(\mathrm{t})) \mathbf{r}, \quad \mathbf{y}=\mathbf{C}(\rho(\mathrm{t})) \mathbf{x}+\mathbf{D}(\rho(\mathrm{t})) \mathbf{r}
$$

The dynamic properties change with $\rho(t)$ but, provided that the rate of change is not too rapid, then the dynamic properties of the time-varying controller, (1), are similar to those of the linear controllers obtained by 'freezing' the value of $\rho$; that is, the nonlinear controller inherits the dynamic properties of the family of linear controllers (see, for example, Desoer 1969). It is noted that there are no direct restrictions on the state, $\mathbf{x}$, or the input, $\mathbf{r}$. The only restriction is on the rate of change of the scheduling variable; indeed, when the scheduling variable is constant, the controller is linear. However, matters are not so simple when the scheduling variable is a function of internal quantities. In this paper, consideration is restricted to the latter.

\subsection{Linear synthesis}

In the gain-scheduling design approach, the (usually nonlinear) plant dynamics are approximated, locally to a specific equilibrium operating point, by a series expansion linearisation. A linear time-invariant controller is then designed which ensures appropriate closed-loop performance when employed with the plant linearisation. This process is repeated for a set of equilibrium operating points, covering the envelope of operation, whilst ensuring that the linear controller designs have compatible structures; for example, when a smoothly gain-scheduled controller is required, the linear controller designs are selected to permit smooth interpolation, in some appropriate manner, between the designs.

At any equilibrium operating point of the plant, all of the plant inputs, including the controller output $\mathbf{y} \in \mathfrak{R}^{\mathrm{p}}$, and all of the plant outputs, including the controller input $\mathbf{r} \in \mathfrak{R}^{\mathrm{m}}$, are constant. It is assumed that the equilibrium operating points of the plant may be minimally parameterised by a quantity, $\pi \in \mathfrak{R}^{\mathrm{q}}$. Consequently, both the equilibrium operating points of the gain-scheduled controller and the local linear time-invariant controllers may also be parameterised by $\pi$. Hence, there exists a family, $\left(\mathbf{r}_{\mathrm{o}}(\pi), \mathbf{y}_{\mathrm{o}}(\pi)\right)$, of constant controller inputs and outputs and a family of linear time-invariant controllers,

$$
\begin{aligned}
& \delta \mathbf{x}_{\pi}=\mathbf{A}_{\pi} \delta \mathbf{x}_{\pi}+\mathbf{B}_{\pi} \delta \mathbf{r}_{\pi}, \quad \delta \mathbf{y}_{\pi}=\mathbf{C}_{\pi} \delta \mathbf{x}_{\pi}+\mathbf{D}_{\pi} \delta \mathbf{r}_{\pi} \\
& \delta \mathbf{r}_{\pi}=\mathbf{r}-\mathbf{r}_{\mathbf{0}}(\pi), \quad \mathbf{y}=\mathbf{y}_{\mathbf{0}}(\pi)+\delta \mathbf{y}_{\pi}
\end{aligned}
$$

corresponding to the set of plant equilibrium operating points. Clearly, (2a) is not unique and the states, $\delta \mathbf{x}_{\pi}$, may be subject to a non-singular linear transformation (which may be different for each equilibrium operating point) without changing the dynamics. In addition, it is assumed that the degrees of the input, output and state are not dependent on $\pi$ and that the matrices, $\mathbf{A}_{\pi}, \mathbf{B}_{\pi}, \mathbf{C}_{\pi}$ and $\mathbf{D}_{\pi}$ are continuous with respect to $\pi$.

\subsection{Nonlinear realisation}

In addition to the synthesis of a family of linear controllers, the gain-scheduling design approach requires the determination of a suitable nonlinear controller; that is, the determination of an appropriate realisation. It is this step which receives consideration in the present paper. Nonlinear gain-scheduled controller realisations of the form,

$$
\dot{\mathbf{x}}=\mathbf{F}(\mathbf{x}, \mathbf{r}), \quad \mathbf{y}=\mathbf{G}(\mathbf{x}, \mathbf{r})
$$

are considered, where $\mathbf{F}(\cdot, \cdot)$ and $\mathbf{G}(\cdot, \cdot)$ are continuous and differentiable, and $\mathbf{x} \in \mathfrak{R}^{\mathrm{n}}$ denotes the state of the controller. The set of equilibrium operating points of the nonlinear controller, (3), consists of those points, $\left(\mathbf{x}_{\mathbf{0}}, \mathbf{y}_{\mathbf{0}}, \mathbf{r}_{\mathbf{0}}\right)$, for which

$$
\mathbf{F}\left(\mathbf{x}_{\mathbf{0}}, \mathbf{r}_{\mathbf{0}}\right)=0, \quad \mathbf{y}_{\mathbf{0}}=\mathbf{G}\left(\mathbf{x}_{\mathbf{0}}, \mathbf{r}_{\mathbf{0}}\right)
$$

Let $\Phi: \Re^{\mathrm{n}} \times \mathfrak{R}^{\mathrm{m}}$ denote the space consisting of the union of the state, $\mathbf{x}$, with the input, $\mathbf{r}$. The locus of the set of equilibrium operating points of the nonlinear controller, $\left(\mathbf{x}_{\mathbf{0}}, \mathbf{r}_{\mathbf{0}}\right)$, forms a surface in $\Phi$ and the response of the controller to a general time-varying input, $\mathbf{r}(\mathrm{t})$, is depicted by a trajectory in $\Phi$.

Assume that (4) is suitably invertible so that the equilibrium values of the state, $\mathbf{x}_{\mathbf{0}}$, may be determined from the equilibrium values of the controller input, $\mathbf{r}_{\mathbf{0}}$, and output, $\mathbf{y}_{\mathbf{0}}$. This assumption ensures that the equilibrium characteristics are independent of the choice of controller state. For consistency with section 2.1, the set of equilibrium operating points, $\left(\mathbf{r}_{\mathbf{0}}, \mathbf{y}_{\mathbf{0}}\right)$, must be the same as the family $\left(\mathbf{r}_{\mathbf{0}}(\pi), \mathbf{y}_{\mathbf{0}}(\pi)\right)$ and the surface of equilibrium operating points in $\Phi$ must be of dimension q. The quantity, $\pi$, provides an index for the equilibrium operating points which is independent 
of the realisation whereas the points $\left(\mathbf{x}_{\mathbf{0}}, \mathbf{r}_{\mathbf{0}}\right)$ themselves, and the mapping from $\pi$ to $\left(\mathbf{x}_{\mathbf{0}}, \mathbf{r}_{\mathbf{0}}\right)$ and the inverse mapping from $\left(\mathbf{x}_{\mathbf{0}}, \mathbf{r}_{\mathbf{0}}\right)$ to $\pi$, are dependant on the choice of realisation, (3). The scheduling variable is a function, $\rho:\left(\mathbf{x}_{\mathbf{0}}, \mathbf{r}_{\mathbf{0}}\right) \mapsto \Re^{q}$, which is an isomorphism of the inverse mapping from $\left(\mathbf{x}_{\mathbf{0}}, \mathbf{r}_{\mathbf{0}}\right)$ to the parameterisation of the equilibrium operating points. Hence, the set of equilibrium operating points for the nonlinear controller, (3), can be parameterised with respect to $\rho$; namely, $\left(\mathbf{x}_{\mathbf{0}}(\rho), \mathbf{y}_{\mathbf{o}}(\rho), \mathbf{r}_{\mathbf{0}}(\rho)\right)$. The domain of $\rho$ is usually not confined to the set of equilibrium operating points, $\left(\mathbf{x}_{\mathbf{0}}, \mathbf{r}_{\mathbf{0}}\right)$ : at a general operating point, $(\mathbf{x}, \mathbf{r}), \rho:(\mathbf{x}, \mathbf{r}) \mapsto \mathfrak{R}^{\mathrm{q}}$ such that $\rho(\mathbf{x}, \mathbf{r})=\rho\left(\mathbf{x}_{\mathbf{0}}, \mathbf{r}_{\mathbf{o}}\right)$ for some $\left(\mathbf{x}_{\mathbf{0}}, \mathbf{r}_{\mathbf{o}}\right)$. The scheduling variable, thereby, indicates some member of the linear family, (2), at any time, regardless of whether the system is in equilibrium. In this paper, it is assumed that $\rho$ is a continuous function; that is, the discussion here excludes non-smooth scheduling such as switching. Typically, the selection of an appropriate scheduling variable is based on physical insight.

The nonlinear controller, (3), is required to have, in some sense, similar dynamic properties to the members of the linear family, (2), for the widest possible range of operating conditions. Initially, consider the case where the controller is operating locally to a specific equilibrium operating point, $\left(\mathbf{x}_{\mathbf{o}}\left(\rho_{\mathbf{o}}\right), \mathbf{r}_{\mathbf{o}}\left(\rho_{\mathbf{o}}\right)\right)$. There exists a class, $C_{\rho_{o}}$, of inputs and initial conditions for the state, for which the nonlinear controller and the associated member of the linear family are judged to have similar dynamic behaviour. (The definition of the criteria, by which the similarity of dynamic behaviour is assessed, is dependent on the context, in which the requirement for a gain-scheduled controller arises, and on the nature of the controllers). Let the class, $C$, be the union over the $\rho_{\mathrm{o}}$ of the classes $C_{\rho_{o}}$. Now consider the case where the controller is not confined to operating locally to some specific equilibrium operating point. There exists a class, $C_{N}$, of inputs and initial conditions for the state such that the response of the nonlinear controller, whilst in the vicinity of a particular equilibrium operating point, is judged, in some contextually appropriate sense, to have similar dynamic behaviour to the corresponding member of the family of linear controllers. The classes, $C$ and $C_{N}$, are non-empty since they contain the equilibrium values $\mathbf{r}_{\mathbf{0}}\left(\rho_{\mathbf{0}}\right)$ and $\mathbf{x}_{\mathbf{0}}\left(\rho_{\mathbf{0}}\right)$. The definitions of the criteria, by which the similarity of dynamic behaviour is assessed in the two cases, are assumed to be such that, for a sequence of nonlinear realisations for a family of linear controllers, the trend is for the membership of the class $C_{N}$ to increase as the membership of the class $C$ increases. This assumption is not unnatural since the membership of the classes is a reflection of the "strength of the controller nonlinearity" by whatever means it is defined.

It should be noted that the relationship between the equilibrium input and output of the controller, $\mathbf{r}_{\mathbf{0}}(\bullet)$ and $\mathbf{y}_{\mathbf{0}}(\bullet)$, can have a considerable impact on the nonlinear controller design. Whilst this issue is largely neglected in the literature, it is clear that a strong restriction is imposed on the class of allowable nonlinear controllers; for example, the functions, $\mathbf{F}(\bullet, \bullet)$ and $\mathbf{G}(\bullet, \bullet)$, in (3) are not independent since,

$\mathbf{F}\left(\mathbf{x}_{\mathbf{0}}(\rho), \mathbf{r}_{\mathbf{0}}(\rho)\right)=0, \mathbf{G}\left(\mathbf{x}_{\mathbf{0}}(\rho), \mathbf{r}_{\mathbf{0}}(\rho)\right)=\mathbf{y}_{\mathbf{0}}(\rho)$

must be jointly satisfied and, when $\mathbf{F}(\bullet, \bullet)$ is invertible so that $\mathbf{x}_{\mathbf{0}}(\bullet)$ is determined by $\mathbf{r}_{\mathbf{0}}(\bullet)$, it follows that the output function, $\mathbf{G}(\bullet, \bullet)$, is, essentially, completely specified by the equilibrium input/output relationship and the choice of $\mathbf{F}(\bullet, \bullet)$. It is possible to satisfy the relationship between the equilibrium input and output, whilst maintaining design freedom, by including explicit functions for $\mathbf{r}_{\mathbf{o}}(\bullet)$ and $\mathbf{y}_{\mathbf{0}}(\bullet)$ within the controller (Shamma \& Athans 1990, Lawrence \& Rugh 1995). However, the derivation of the explicit functions is extremely onerous and quite undesirable in most applications. Unless otherwise stated, it is assumed that the controllers discussed below are compatible with the equilibrium input/output conditions.

\subsection{Previous approaches}

Perhaps the most common approach to realising a gain-scheduled controller is to simply substitute a scheduling variable, $\rho(\mathbf{x}, \mathbf{r})$, for $\pi$ in the family of local linear controllers, (2), to obtain a nonlinear controller. When the scheduling variable, $\rho$, varies sufficiently slowly (trivially, when the rate of variation is zero), the dynamic characteristics of the linear family are inherited by the nonlinear controller. However, in the nonlinear controller the scheduling variable, $\rho$, varies with the input and/or the state whilst in the local linear controller, (2), it is fixed. Hence, in general, the series expansion linearisation of the nonlinear controller about an equilibrium operating point, at which the scheduling variable has the value $\rho_{\mathbf{0}}$, contains terms, related to the perturbations, $\delta \rho$, in the scheduling variable, not present in the linear family, (2) (where $\delta \rho$ equals $\rho-\rho_{\mathbf{0}}$ ). Consequently, for the nonlinear controller to have similar dynamics to the linear family, it is necessary to impose a constraint on the variation of $\rho$; namely, that the first order perturbations in $\delta \rho$, and so $\delta \mathbf{x}$ and $\delta \mathbf{r}$, must be negligible. This constraint applies even when the state is confined to a small neighbourhood about a single equilibrium operating point which is clearly not a priori necessary. Hence, for this class of nonlinear controller the constraints imposed on $\rho$ seem unnecessarily strict.

As an alternative, Lawrence \& Rugh (1995) (and also Kaminer et al. 1995, in a more restrictive context) propose that the nonlinear controller should be selected to ensure that its series expansion linearisation at each equilibrium operating point corresponds to the appropriate member of the linear family, (2). In other words, within the 
neighbourhood, about a specific equilibrium operating point, for which the second order perturbations in $\delta \mathbf{x}$ and $\delta \mathbf{r}$ are negligible, the nonlinear controller is required to have similar dynamics to the appropriate member of the linear family. The nonlinear controller, therefore, has the required dynamic behaviour, locally to each equilibrium operating point, for a less strict constraint on $\delta \mathbf{x}$ and $\delta \mathbf{r}$ than the previous approach.

\section{Selection criteria for the realisation of gain-scheduled controllers}

It is clearly attractive to require that the controllers are consistent with the approach of Lawrence \& Rugh (1995) since the series expansion linearisation of the controller, at each equilibrium operating point, then corresponds to the appropriate member of the linear family, (2). However, there exist infinitely many nonlinear controllers satisfying the local linear equivalence condition of Lawrence \& Rugh for a particular family of linear controllers. The size of the classes, $C_{\rho_{o}}$, of inputs and initial conditions may vary greatly for different choices of controller realisation and may, in fact, be relatively large for some choices but vanishingly small for others. Since the size of the classes of valid inputs and initial conditions is variable and may be unnecessarily restricted by the choice of realisation adopted, the requirement of local linear equivalence is, by itself, an inadequate guide to the choice of a minimally nonlinear controller realisation; that is, a realisation for which the restrictions on the inputs and the initial values of the states are minimal (Leith \& Leithead 1997a). A criterion is, therefore, required which provides guidance to those realisations satisfying local linear equivalence that do not unnecessarily diminish the class of valid inputs and initial conditions.

\subsection{Extended local linear equivalence}

The extent to which the dynamics vary over the locus of equilibrium operating points, being defined by the family of linear controllers, (2), is clearly inherent to the gain-scheduled controller. However, the extent to which the dynamics vary as the operating point is displaced away from the locus of equilibrium operating points is, in contrast, dependent on the controller realisation. Restrictions are imposed on the inputs and initial conditions by both aspects of the controller nonlinearity. The restriction imposed by the first aspect is somewhat similar to that encountered in exogenously gainscheduled controllers; roughly speaking, the inputs and initial conditions are restricted to prevent the locus of equilibrium operating points from being traversed too rapidly thereby preventing the dynamic properties, which vary over the locus of equilibrium operating points, from varying too rapidly. When the inputs and initial conditions are such that the variation in the scheduling variable is small and consideration may be confined to a single member of the linear family, this restriction does not apply. However, the second aspect still imposes a restriction on the inputs and initial conditions. The restriction imposed by the second aspect is, evidently, additional to and, in some sense, independent of the first.

The nonlinear controller, (3), may be reformulated as,

$$
\begin{aligned}
& \delta \mathbf{x}=\nabla_{\mathbf{x}} \mathbf{F}\left(\mathbf{x}_{\mathbf{0}}\left(\rho_{\mathbf{0}}\right), \mathbf{r}_{\mathbf{0}}\left(\rho_{\mathbf{o}}\right)\right) \delta \mathbf{x}+\nabla_{\mathbf{r}} \mathbf{F}\left(\mathbf{x}_{\mathbf{0}}\left(\rho_{\mathbf{0}}\right), \mathbf{r}_{\mathbf{o}}\left(\rho_{\mathbf{0}}\right)\right) \delta \mathbf{r}+\varepsilon_{\mathbf{F}} \\
& \delta \mathbf{y}=\nabla_{\mathbf{x}} \mathbf{G}\left(\mathbf{x}_{\mathbf{0}}\left(\rho_{\mathbf{o}}\right), \mathbf{r}_{\mathbf{0}}\left(\rho_{\mathbf{0}}\right)\right) \mathbf{x}+\nabla_{\mathbf{r}} \mathbf{G}\left(\mathbf{x}_{\mathbf{0}}\left(\rho_{\mathbf{0}}\right), \mathbf{r}_{\mathbf{0}}\left(\rho_{\mathbf{o}}\right)\right) \delta \mathbf{r}+\varepsilon_{\mathbf{G}} \\
& \delta \mathbf{r}=\mathbf{r}-\mathbf{r}_{\mathbf{0}}\left(\rho_{\mathbf{0}}\right), \quad \mathbf{y}=\mathbf{y}_{\mathbf{0}}\left(\rho_{\mathbf{0}}\right)+\delta \mathbf{y}
\end{aligned}
$$

where,

$$
\begin{aligned}
& \varepsilon_{\mathbf{F}}=\mathbf{F}(\mathbf{x}, \mathbf{r})-\nabla_{\mathbf{x}} \mathbf{F}\left(\mathbf{x}_{\mathbf{0}}\left(\rho_{\mathbf{o}}\right), \mathbf{r}_{\mathbf{o}}\left(\rho_{\mathbf{o}}\right)\right) \delta \mathbf{x}-\nabla_{\mathbf{r}} \mathbf{F}\left(\mathbf{x}_{\mathbf{0}}\left(\rho_{\mathbf{0}}\right), \mathbf{r}_{\mathbf{o}}\left(\rho_{\mathbf{0}}\right)\right) \delta \mathbf{r} \\
& \varepsilon_{\mathbf{G}}=\mathbf{G}(\mathbf{x}, \mathbf{r})-\mathbf{G}\left(\mathbf{x}_{\mathbf{0}}, \mathbf{r}_{\mathbf{0}}\right)-\nabla_{\mathbf{x}} \mathbf{G}\left(\mathbf{x}_{\mathbf{0}}\left(\rho_{\mathbf{0}}\right), \mathbf{r}_{\mathbf{o}}\left(\rho_{\mathbf{0}}\right)\right) \delta \mathbf{x}-\nabla_{\mathbf{r}} \mathbf{G}\left(\mathbf{x}_{\mathbf{0}}\left(\rho_{\mathbf{0}}\right), \mathbf{r}_{\mathbf{0}}\left(\rho_{\mathbf{0}}\right)\right) \delta \mathbf{r} \\
& \mathbf{x}=\mathbf{x}_{\mathbf{0}}\left(\rho_{\mathbf{0}}\right)+\delta \mathbf{x}
\end{aligned}
$$

for any $\rho_{\mathbf{0}}$. The local linear equivalence requirement is satisfied at an equilibrium operating point, at which $\rho$ has the value, $\rho_{\mathbf{0}}$, provided the derivatives $\nabla_{\mathbf{x}} \mathbf{F}, \nabla_{\mathbf{r}} \mathbf{F}, \nabla_{\mathbf{x}} \mathbf{G}$ and $\nabla_{\mathbf{r}} \mathbf{G}$ match, within a non-singular transformation of the states, the matrices, $\mathbf{A}_{\pi}, \mathbf{B}_{\pi}, \mathbf{C}_{\pi}$ and $\mathbf{D}_{\pi}$, of the appropriate member of the family of linear controllers.

When assessing whether the dynamic behaviour of the nonlinear controller, (3), is locally similar to a particular member of the family of linear controllers, it may naturally be assumed that the criteria employed imposes a restriction on the magnitude of the residuals, $\varepsilon_{\mathbf{F}}$ and $\varepsilon_{\mathbf{G}}$. In general, the residuals may be dependent on every element of $\delta \mathbf{x}$ and $\delta \mathbf{r}$ and, therefore, a restriction is imposed on the magnitude of every element. However, many of these restrictions are not a priori necessary. The inherent nonlinear character of the gain-scheduled controller is embodied in the variation between the members of the family of linear controllers, (2). It follows that the gain-scheduled controller is inherently nonlinear with respect to the scheduling variable, $\rho$, and a particular member of the family of linear controllers is inherently only valid when the perturbation, $\delta \rho$, is restricted to some range which is dependent on the properties of the family of linear controllers. To minimise the restrictions on the perturbations in the elements of $(\mathbf{x}, \mathbf{r})$, it is, therefore, quite natural to require that the residuals depend purely on $\delta \rho$ alone; that is, on $\rho$ and no other combinations of the element. Hence, the nonlinearity must purely be a function of $\rho$. Moreover, since $\rho$ minimally parameterises the family of linear controllers, it is clear that this is the weakest functional dependence of the residuals possible. 
When the controller nonlinearity is purely a function of $\rho$, the nonlinear controller, (3), must be of the form,

$$
\mathbf{x}=\mathbf{A x}+\mathbf{B r}+\mathbf{f}(\rho), \quad \mathbf{y}=\mathbf{C} \mathbf{x}+\mathbf{D r}+\mathbf{g}(\rho)
$$

where $\mathbf{A}, \mathbf{B}, \mathbf{C}, \mathbf{D}$ are constant matrices, $\mathbf{f}(\bullet)$ and $\mathbf{g}(\bullet)$ are differentiable nonlinear functions, and $\nabla_{\mathbf{x}} \rho, \nabla_{\mathrm{r}} \rho$ are functions of $\rho$ alone. The scheduling variable, $\rho(\mathbf{x}, \mathbf{r}) \in \mathfrak{R}^{\mathrm{q}}$, equals the constant value, $\rho_{\mathbf{o}}$, upon a surface of co-dimension $q$ in $\Phi$ and $\nabla_{\mathrm{x}} \rho$ and $\nabla_{\mathrm{r}} \rho$ are constant over each surface. Hence, the normal to each surface is identical at every point on the surface and each surface is, therefore, affine. Moreover, to ensure that $\rho$ is a unique function of $\mathbf{x}$ and $\mathbf{r}$, these surfaces must be parallel for all $\rho$. Consequently, it may be assumed, without loss of generality, that $\nabla_{\mathbf{x}} \rho$ and $\nabla_{\mathbf{r}} \rho$ are constant and $\rho$ is a linear combination of the elements of the state and input. The controller dynamics are linear in those combinations of the elements of $(\mathbf{x}, \mathbf{r})$ that are linearly independent of $\rho$.

The linearisation of the nonlinear controller, (6), at a specific equilibrium operating point, $\left(\mathbf{x}_{\mathbf{0}}\left(\rho_{\mathbf{0}}\right), \mathbf{r}_{\mathbf{0}}\left(\rho_{\mathbf{0}}\right)\right)$, is,

$$
\begin{aligned}
& \delta \mathbf{x}=\left(\mathbf{A}+\nabla_{\mathrm{p}} \mathbf{f}\left(\rho_{\mathbf{o}}\right) \nabla_{\mathrm{x}} \rho\right) \delta \mathbf{x}+\left(\mathbf{B}+\nabla_{\mathrm{p}} \mathbf{f}\left(\rho_{\mathrm{o}}\right) \nabla_{\mathrm{r}} \rho\right) \delta \mathbf{r} \\
& \delta \mathbf{y}=\left(\mathbf{C}+\nabla_{\mathrm{\rho}} \mathbf{g}\left(\rho_{\mathbf{o}}\right) \nabla_{\mathbf{x}} \rho\right) \delta \mathbf{x}+\left(\mathbf{D}+\nabla_{\mathrm{p}} \mathbf{g}\left(\rho_{\mathbf{0}}\right) \nabla_{\mathrm{r}} \rho\right) \delta \mathbf{r}
\end{aligned}
$$

Unnecessary restrictions on the membership of the classes, $C_{\rho_{o}}$, of inputs and initial conditions for the state, for which the linearisations are locally valid, are avoided by the realisation, (6); in this sense, the size of the classes, $C_{\rho_{o}}$ and so $C$, is maximised. Since the membership of the class, $C_{N}$, tends to increase as the membership of the class, $C$, increases, membership of $C_{N}$ is, in general, greater for a controller with the realisation (6), i.e. satisfying the so-called extended local linear equivalence condition, than for a controller satisfying the local linear equivalence condition of Lawrence \& Rugh (1995). The restriction imposed on the class of inputs and initial conditions by the extent, to which the dynamics vary as the operating point is displaced away from the locus of equilibrium operating points, is completely relaxed for the realisations satisfying the extended local linear equivalence condition. Only the inherent restriction imposed on the class by the extent to which the dynamics vary over the locus of equilibrium operating points remains in place.

The formulation, (6), is equivalent, in a SISO context, to the extended local linear equivalence condition proposed by Leith \& Leithead $(1994,1996)$.

\subsubsection{Linearisation at non-equilibrium operating points}

For consistency with the restriction on the magnitude of the residuals imposed by the requirement that the dynamic behaviour of the nonlinear controller, (3), is locally similar to a particular member of the family of linear controllers, the state, $\delta \mathbf{x}$, of the nonlinear controller, (5), and the input, $\delta \mathbf{r}$, must belong to some neighbourhood of $\left(\mathbf{x}_{\mathbf{0}}\left(\rho_{\mathbf{0}}\right), \mathbf{r}_{\mathbf{0}}\left(\rho_{\mathbf{0}}\right)\right.$ within which they are sufficiently small that $\varepsilon_{\mathbf{F}}$ and $\varepsilon_{\mathbf{G}}$ are negligible. (Alternatively, the state of the linear controller, obtained from (5) by setting $\varepsilon_{\mathbf{F}}$ and $\varepsilon_{\mathbf{G}}$ to zero, and the input could be required to belong to the neighbourhood. Although the class, for which this latter requirement is met, is not identical to $C_{\rho_{o}}$, it is not substantially different. When comparing the membership of the class of inputs and initial conditions for different realisations, this alternative requirement has the advantage that the state does not change with the realisation since the linearisations are the same). The situation for a controller satisfying the local linear equivalence condition of Lawrence \& Rugh (1995) is illustrated in figure 1 for a SISO first-order controller whilst the situation for a controller satisfying the extended local linear equivalence condition, is illustrated in figure 2 : the shaded regions notionally indicate the neighbourhoods within which the $\delta \mathbf{x}$ and $\delta \mathbf{r}$ are sufficiently small that $\varepsilon_{\mathbf{F}}$ and $\varepsilon_{\mathrm{G}}$ are negligible and a particular linearisation is valid. It should be noted that the neighbourhoods for the realisation, (6), are infinite in extent, having no a priori restriction in the directions in which $\rho$ is constant. Irrespective of any difference in scaling of the states, the neighbourhoods in figure 2 are significantly larger than the neighbourhoods in figure 1.

The nonlinear controller, (6), can be linearised at any operating point, including non-equilibrium operating points, for which the value of $\rho$ is within its domain; in many cases at any operating point in the space, $\Phi$. Any point, for which $\rho$ has the value $\rho_{\mathbf{0}}$, is in the neighbourhood associated with the equilibrium operating point for that value of $\rho_{\mathbf{0}}$. Hence, at a non-equilibrium operating point the nonlinear controller can be linearised by associating it with the linear controller at the equilibrium point for which the value of $\rho$ is the same; that is, at a point for which the scheduling variable has the value $\rho_{0}$, the linearisation of (6) is (7). (Note, the linearisation is not obtained by perturbing the system about the non-equilibrium operating point and neglecting the inhomogeneous term, although that would result in a similar description for those systems satisfying the extended local linear equivalence condition). This linearisation is valid in any neighbourhood of the non-equilibrium operating point which is contained within the neighbourhood associated with the corresponding equilibrium operating point. Hence, the extended local linear equivalence condition can be interpreted as requiring that the linearisation of the nonlinear controller corresponds to the appropriate member of the linear family at all operating points and not just the equilibrium operating points as required by the local linear equivalence condition of Lawrence \& Rugh (1995). 


\subsection{Example}

The foregoing points are illustrated by the following example. Suppose that the family of second-order SISO linear controllers,

$$
\delta \mathbf{x}=\mathbf{A}_{\pi} \delta \mathbf{x}+\mathbf{B}_{\pi} \delta \mathrm{r}, \quad \delta \mathrm{y}=\mathbf{C}_{\pi} \delta \mathbf{x}+\mathbf{D}_{\pi} \delta \mathrm{r}
$$

where $\delta \mathbf{x}=\left[\delta \mathrm{x}_{1} \delta \mathrm{x}_{2}\right]^{\mathrm{T}}$

$$
\mathbf{A}_{\pi}=\left[\begin{array}{cc}
0 & 0 \\
(1-\mathrm{ab}) \mathrm{g}_{\pi} & -\mathrm{a}
\end{array}\right], \quad \mathbf{B}_{\pi}=\left[\begin{array}{l}
1 \\
0
\end{array}\right], \mathbf{C}_{\pi}=\left[\begin{array}{ll}
\operatorname{bg}_{\pi} & 1
\end{array}\right], \mathbf{D}_{\pi}=0
$$

has been designed, where $\mathrm{g}_{\pi}$ varies as a continuous function of the equilibrium operating point and is non-zero. The transfer function of the member of the family associated with $\pi$ equal to $\pi_{\mathrm{o}}$ is,

$$
Y(s)=g_{\pi_{0}} \frac{(b s+1)}{s(s+a)} R(s)
$$

where $\mathrm{Y}(\mathrm{s}), \mathrm{R}(\mathrm{s})$ are the Laplace transforms of, respectively, $\delta \mathrm{y}(\mathrm{t})$ and $\delta \mathrm{r}(\mathrm{t})$. The values of $\mathrm{g}_{\pi}$ are assumed to be finite and either strictly positive or strictly negative; that is, there exist $a_{1}$ and $a_{2}$ such that

$$
0<\mathrm{a}_{1} \leq \mathrm{g}_{\pi} \leq \mathrm{a}_{2}<\propto \quad \text { or } \quad-\propto<\mathrm{a}_{1} \leq \mathrm{g}_{\pi} \leq \mathrm{a}_{2}<0
$$

The output, $y$, is selected as the scheduling variable on the assumption that the equilibrium operating points of the controller can be parameterised by y and that there exists a continuous function,

$$
\mathrm{g}(\bullet): \mathfrak{R} \rightarrow\left\{\mathrm{g}_{\pi}\right\} \text {. }
$$

(Owing to the pure integral action present in the members of the family, (8), the input to the controller must be zero at an equilibrium operating point and the locus of equilibrium operating points cannot, therefore, be parameterised by the input).

\section{$\underline{\text { Realisation A }}$}

Consider the nonlinear controller obtained by simply replacing $\mathrm{g}_{\pi}$ in (8) by the function $\mathrm{g}(\mathrm{y})$ as depicted in figure $3 \mathrm{a}$. Such an approach is widely employed to construct gain-scheduled controllers. The dynamics of the nonlinear controller are described by

where $\mathbf{x}=\left[\begin{array}{ll}\mathrm{x}_{1} & \mathrm{x}_{2}\end{array}\right]^{\mathrm{T}}$

$$
\mathbf{x}=\mathbf{A}_{\mathrm{a}} \mathbf{x}+\mathbf{B}_{\mathrm{a}} \mathrm{r}+\mathbf{f}_{\mathrm{a}}(\mathbf{x}, \mathrm{r}), \mathrm{y}=\mathbf{C}_{\mathrm{a}} \mathbf{x}+\mathbf{D}_{\mathrm{a}} \mathrm{r}+\mathrm{g}_{\mathrm{a}}(\mathbf{x}, \mathrm{r})
$$

$$
\mathbf{A}_{\mathrm{a}}=\left[\begin{array}{cc}
0 & 0 \\
0 & -\mathrm{a}
\end{array}\right], \quad \mathbf{B}_{\mathrm{a}}=\left[\begin{array}{l}
1 \\
0
\end{array}\right], \mathbf{C}_{\mathrm{a}}=\left[\begin{array}{ll}
0 & 1
\end{array}\right], \mathbf{D}_{\mathrm{a}}=0, \mathbf{f}_{\mathrm{a}}(\mathbf{x}, \mathrm{r})=\left[\begin{array}{c}
0 \\
(1-\mathrm{ab}) \mathrm{g}(\mathrm{y}(\mathbf{x})) \mathrm{x}_{1}
\end{array}\right], \quad \mathrm{g}_{\mathrm{a}}(\mathbf{x}, \mathrm{r})=\mathrm{bg}(\mathrm{y}(\mathbf{x})) \mathrm{x}_{1}
$$

and $y(\mathbf{x})$ is a solution to the implicit nonlinear equation,

$$
\mathrm{y}=\mathrm{x}_{2}+\operatorname{bg}(\mathrm{y}) \mathrm{x}_{1}
$$

From assumptions (10) and (11), the straight lines of constant $\mathrm{y},(12 \mathrm{c})$, cover the complete $\left(\mathrm{x}_{1}, \mathrm{x}_{2}\right)$ plane. Hence, there exists at least one value of $y$ satisfying (12c) for all $x_{1}$ and $x_{2}$. When more than one value exists, the appropriate one is determined by continuity. The initial condition for the state, $\mathbf{x}$, must be accompanied by an appropriate, context dependent, choice of $y$. The locus of equilibrium operating points is the set of points, $\left(a y_{0} / g\left(y_{0}\right),(1-a b) y_{0}, 0\right),-\propto<y_{o}<\propto$, and the scheduling variable, $\mathrm{y}$, is constant on the planes $\mathrm{x}_{2}+\mathrm{bg}(\mathrm{y}) \mathrm{x}_{1}=\mathrm{y}_{\mathrm{o}}$. Locally to an equilibrium operating point, at which the nominal value of $\mathrm{y}$ is $\mathrm{y}_{\mathrm{o}}$,

$$
\mathrm{r}=0+\delta \mathrm{r}, \quad \mathrm{x}_{1}=\mathrm{x}_{1 \mathrm{o}}+\delta \mathrm{x}_{1}, \mathrm{x}_{2}=\mathrm{x}_{2 \mathrm{o}}+\delta \mathrm{x}_{2}, \mathrm{y}=\mathrm{y}_{\mathrm{o}}+\delta \mathrm{y}
$$

and the nonlinear controller, (12), can be reformulated as

where

$$
\begin{aligned}
& \delta \mathbf{x}=\left[\begin{array}{cc}
0 & 0 \\
(1-a b) g\left(y_{o}\right) & -a
\end{array}\right] \delta \mathbf{x}+\left[\begin{array}{l}
1 \\
0
\end{array}\right] \delta r+\varepsilon_{x} \\
& \delta y=\left[b g\left(y_{o}\right) 1\right] \delta \mathbf{x}+\varepsilon_{y}
\end{aligned}
$$

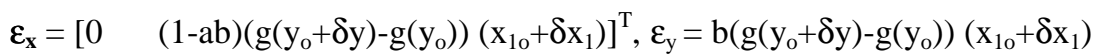

The linear system obtained by setting $\varepsilon_{\mathrm{x}}$ and $\varepsilon_{\mathrm{y}}$ to zero in (14) is the same as (8) as required. For the linearisation to be locally valid, $\delta \mathrm{x}_{1}$ and $\delta \mathrm{y}$ must be sufficiently small that $\varepsilon_{\mathrm{x}}$ and $\varepsilon_{\mathrm{y}}$ are negligible. It should be noted that, as $\mathrm{y}_{\mathrm{o}}$ varies and the magnitude of $\mathrm{x}_{1 \mathrm{o}}$ increases, the membership of the class, $C_{\rho_{o}}$, rapidly diminishes until it becomes, in terms of the context, insignificant. This is a consequence of the realisation not satisfying the local linear equivalence condition, with 
the result that first order perturbations in $\delta y$ are present in the expressions for $\varepsilon_{\mathrm{x}}$ and $\varepsilon_{\mathrm{y}}$. Unless the operational envelope of the controller is confined to the vicinity of the equilibrium operating points for which the magnitude of $\mathrm{x}_{10}$ is restricted, $\varepsilon_{\mathrm{x}}$ and $\varepsilon_{\mathrm{y}}$ can be arbitrarily large for some $\mathrm{x}_{1 \mathrm{o}}$ for any non-zero values of $\delta \mathrm{y}$.

\section{$\underline{\text { Realisation B }}$}

An alternative nonlinear controller, which employs a velocity formulation similar to that proposed by Kaminer $e t$ al. (1995) (and with the integral-error form discussed by Lawrence \& Rugh 1995), is shown in figure 3b. The dynamics of the controller are described by

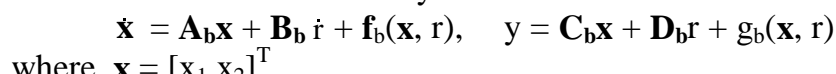

$$
\mathbf{A}_{\mathrm{b}}=\left[\begin{array}{cc}
-\mathrm{a} & 0 \\
1 & 0
\end{array}\right], \quad \mathbf{B}_{\mathrm{b}}=0, \mathbf{C}_{\mathrm{b}}=\left[\begin{array}{ll}
0 & 1
\end{array}\right], \mathbf{D}_{\mathrm{b}}=0, \mathbf{f}_{\mathrm{b}}(\mathbf{x}, \mathrm{r})=\left[\begin{array}{c}
\mathrm{g}\left(\mathrm{x}_{2}\right)(1-\mathrm{ab}) \mathrm{r} \\
\mathrm{bg}\left(\mathrm{x}_{2}\right) \mathrm{r}
\end{array}\right], \mathrm{g}_{\mathrm{b}}(\mathbf{x}, \mathrm{r})=0
$$

The locus of equilibrium operating points is the set of points, $\left(0, y_{0}, 0\right),-\propto<y_{0}<\propto$, and the scheduling variable, $y$, is constant on the planes $\mathrm{x}_{2}=\mathrm{y}_{\mathrm{o}}$. Locally to an equilibrium operating point at which the nominal value of $\mathrm{y}$ is $\mathrm{y}_{\mathrm{o}}$,

$$
\mathrm{r}=0+\delta \mathrm{r}, \quad \mathrm{x}_{1}=0+\delta \mathrm{x}_{1}, \quad \mathrm{x}_{2}=0+\delta \mathrm{x}_{2}, \quad \mathrm{y}=\mathrm{y}_{\mathrm{o}}+\delta \mathrm{y}
$$

and the nonlinear dynamics, (15), can be reformulated as

where

$$
\begin{aligned}
& \delta \mathbf{x}=\left[\begin{array}{cc}
-\mathrm{a} & 0 \\
1 & 0
\end{array}\right] \delta \mathbf{x}+\left[\begin{array}{c}
\mathrm{g}\left(\mathrm{y}_{\mathrm{o}}\right)(1-\mathrm{ab}) \\
\mathrm{bg}\left(\mathrm{y}_{\mathrm{o}}\right)
\end{array}\right] \delta \mathrm{r}+\varepsilon_{\mathbf{x}} \\
& \delta \mathbf{y}=\left[\begin{array}{ll}
0 & 1
\end{array}\right] \delta \mathbf{x}
\end{aligned}
$$

$$
\varepsilon_{\mathrm{x}}=\left[(1-\mathrm{ab})\left(\mathrm{g}\left(\mathrm{y}_{\mathrm{o}}+\delta \mathrm{y}\right)-\mathrm{g}\left(\mathrm{y}_{\mathrm{o}}\right)\right) \delta \mathrm{r} \quad \mathrm{b}\left(\mathrm{g}\left(\mathrm{y}_{\mathrm{o}}+\delta \mathrm{y}\right)-\mathrm{g}\left(\mathrm{y}_{\mathrm{o}}\right)\right) \delta \mathrm{r}\right]^{\mathrm{T}}
$$

The linear system obtained by setting $\varepsilon_{\mathbf{x}}$ to zero in (17) is the same as (8) as required. For the linearisation to be valid, $\delta y$ and $\delta \mathbf{r}$ must be sufficiently small that $\varepsilon_{\mathbf{x}}$ is negligible. It should be noted that, as $\mathrm{y}_{\mathrm{o}}$ varies, the membership of the class, $C_{\rho_{o}}$, remains significant. This is a consequence of the nonlinear controller satisfying the local linear equivalence condition. Hence, in general, the classes, $C_{\rho_{o}}$, for realisation $\mathrm{B}$, are larger than the classes, $C_{\rho_{o}}$, for realisation $\mathrm{A}$. Clearly, realisation B is more weakly nonlinear than realisation A. However, it is evident that the controller of figure $3 \mathrm{~b}$ does not satisfy the extended local linear equivalence condition.

\section{$\underline{\text { Realisation C }}$}

Now, consider the nonlinear controller shown in figure 3c. Its dynamics are described by the differential equation $\mathbf{x}=\mathbf{A}_{\mathbf{c}} \mathbf{x}+\mathbf{B}$
where $\mathbf{x}=\left[\begin{array}{ll}\mathrm{x}_{1} & \mathrm{x}_{2}\end{array}\right]^{\mathrm{T}}$,

$$
\mathbf{A}_{\mathrm{c}}=\left[\begin{array}{cc}
-\mathrm{a} & 0 \\
0 & 0
\end{array}\right], \quad \mathbf{B}_{\mathrm{c}}=\left[\begin{array}{c}
1-\mathrm{ab} \\
0
\end{array}\right], \mathbf{C}_{\mathrm{c}}=\left[\begin{array}{ll}
0 & 1
\end{array}\right], \mathbf{D}_{\mathrm{c}}=0, \mathbf{f}_{\mathbf{c}}(\mathbf{x}, \mathrm{r})=\left[\begin{array}{c}
0 \\
\mathrm{~g}\left(\mathrm{x}_{2}\right)\left(\mathrm{x}_{1}+\mathrm{br}\right)
\end{array}\right], \mathrm{g}_{\mathrm{c}}(\mathbf{x}, \mathrm{r})=0
$$

The realisation, (18), does not explicitly have the extended local linear equivalence condition form, (6). However, the controller dynamics, (18), may be reformulated as

$$
\dot{\hat{\mathbf{x}}}=\hat{\mathbf{A}}_{\mathrm{c}} \dot{\hat{\mathbf{x}}}+\dot{\mathbf{B}}_{\mathrm{c}} \mathrm{r}+\hat{\mathbf{f}}_{\mathrm{c}}(\mathbf{x}, \mathrm{r}), \quad \mathrm{y}=\dot{\mathbf{C}}_{\mathrm{c}} \dot{\mathbf{x}}+\hat{\mathbf{D}}_{\mathrm{c}} \mathrm{r}+\dot{\mathrm{g}}_{\mathrm{c}}(\mathbf{x}, \mathrm{r})
$$

where $\hat{\mathbf{x}}=\left[\begin{array}{ll}\mathrm{x}_{1} & \hat{\mathbf{x}}_{2}\end{array}\right]^{\mathrm{T}}$,

$$
\begin{aligned}
& \hat{\mathbf{A}}_{\mathrm{c}}=\left[\begin{array}{cc}
-\mathrm{a} & 0 \\
1 & 0
\end{array}\right], \hat{\mathbf{B}}_{\mathrm{c}}=\left[\begin{array}{c}
1-\mathrm{ab} \\
\mathrm{b}
\end{array}\right], \hat{\mathbf{C}}_{\mathrm{c}}=0, \hat{\mathbf{D}}_{\mathrm{c}}=0, \hat{\mathbf{f}}_{\mathrm{c}}(\mathbf{x}, \mathrm{r})=0, \hat{\mathrm{g}}_{\mathrm{c}}(\mathbf{x}, \mathrm{r})=\mathrm{G}\left(\hat{\mathrm{x}}_{2}\right) \\
& \mathrm{G}^{-1}(\mathrm{y})=\int_{0}^{\mathrm{y}} \frac{1}{\mathrm{~g}(\mathrm{~s})} \mathrm{ds}
\end{aligned}
$$

which explicitly satisfies the extended local linear equivalence condition. (By assumption (10), G(•) exists since $\mathrm{G}^{-1}(\bullet)$ is monotonic and, therefore, invertible). The locus of equilibrium operating points is the set of points $\left(0, \mathrm{y}_{\mathrm{o}}, 0\right)$, $-\propto<\mathrm{y}_{\mathrm{o}}<\propto$, and the scheduling variable, $\mathrm{y}$, is constant on the planes $\hat{\mathrm{x}}_{2}=\mathrm{G}^{-1}\left(\mathrm{y}_{\mathrm{o}}\right)$. Locally to an equilibrium operating point at which the nominal value of $\mathrm{y}$ is $\mathrm{y}_{\mathrm{o}}$,

$$
\mathrm{r}=0+\delta \mathrm{r}, \quad \mathrm{x}_{1}=0+\delta \mathrm{x}_{1}, \quad \hat{\mathrm{x}}_{2}=\hat{\mathrm{x}}_{2 \mathrm{o}}+\delta \hat{\mathrm{x}}_{2}, \mathrm{y}=\mathrm{y}_{\mathrm{o}}+\delta \mathrm{y}
$$

and the nonlinear dynamics, (19), can be reformulated as, 


$$
\begin{aligned}
& \delta \dot{\hat{\mathbf{x}}}=\left[\begin{array}{cc}
-\mathrm{a} & 0 \\
1 & 0
\end{array}\right] \delta \hat{\mathbf{x}}+\left[\begin{array}{c}
1-\mathrm{ab} \\
\mathrm{b}
\end{array}\right] \delta \mathrm{r} \\
& \delta y=\left[\begin{array}{ll}
0 & \mathrm{~g}\left(\mathrm{y}_{\mathrm{o}}\right)
\end{array}\right] \delta \dot{\mathbf{x}}+\varepsilon_{\mathrm{y}}
\end{aligned}
$$

where

$$
\varepsilon_{\mathrm{y}}=-\mathrm{g}\left(\mathrm{y}_{\mathrm{o}}\right)\left[\mathrm{G}^{-1}\left(\mathrm{y}_{\mathrm{o}}+\delta \mathrm{y}\right)-\mathrm{G}^{-1}\left(\mathrm{y}_{\mathrm{o}}\right)-\nabla \mathrm{G}^{-1}\left(\mathrm{y}_{\mathrm{o}}\right) \delta \mathrm{y}\right]
$$

The linear system obtained by setting $\varepsilon_{\mathrm{y}}$ to zero in (21) is the same as (8) as required. For the linearisation to be valid, only $\delta y$ must be sufficiently small that $\varepsilon_{\mathrm{y}}$ is negligible. This is a consequence of the nonlinear controller realisation $\mathrm{C}$ satisfying the extended local linear equivalence condition. In general, the membership of the classes, $C_{\rho_{o}}$, for the realisation $\mathrm{C}$ are larger than the classes, $C_{\rho_{o}}$, for realisation $\mathrm{B}$ since the magnitude of both the input, $\delta$ r, and the output, $\delta y$, are restricted in the latter but only the magnitude of the output, $\delta \mathrm{y}$, is restricted in the former with no a priori restriction on the input, $\delta$. Clearly, realisation $\mathrm{C}$ is more weakly nonlinear than realisation $\mathrm{B}$. The neighbourhoods associated with the equilibrium operating points cover the entire $\left(\mathrm{x}_{1}, \hat{\mathrm{x}}_{2}, \mathrm{r}\right)$ space.

When comparing the three realisations, the classes, $C_{\rho_{o}}$, are sufficiently different that there is no need to make the state space forms of the linear controllers identical by transforming the states.

The foregoing simple example does not directly consider the performance benefits of adopting a gain-scheduled controller that satisfies the extended local linear equivalence condition and which, thereby, has appropriate dynamics for the widest class of inputs and initial conditions. However, these benefits, which must, as usual, be confirmed by analysis and/or simulation studies, can be quite considerable. For example, in the context of wind turbine regulation, Leith \& Leithead (1996) observe that an appropriately realised gain-scheduled controller achieves a substantial improvement in performance in comparison to a well-designed linear controller. However, this performance gain is effectively lost when gain-scheduled controller realisations are employed which satisfy only local linear equivalence about the equilibrium operating points rather than the extended local linear equivalence condition. Consequently, the nature of the realisation adopted plays a central role in attaining the required performance. The context of wind turbine regulation is particularly well suited to assessing the effectiveness of the nonlinear controller in that, even though the plant is fixed, the controller is strongly scheduled; that is, the nonlinearity is strong and the scheduling variable cannot $a$ priori be assumed to be slowly varying.

\subsection{Relaxation of restriction on initial conditions}

In section 3.1, minimally nonlinear controller realisations are defined; namely, those satisfying an extended local linear equivalence condition, (6). For these realisations, the restriction imposed on the class of inputs and initial conditions of the state by the extent, to which the dynamics vary as the operating point is displaced away from the locus of equilibrium operating points, is completely relaxed. Only the restriction imposed on the class of inputs and initial conditions by the extent, to which the dynamics vary over the locus of equilibrium operating points, remains. The latter is essentially a restriction on the rate of variation of the scheduling variable in (6). The response of the system, (6), and so the rate of variation of the scheduling variable depends on the input and initial conditions of the state. In general, the dependence on the initial conditions becomes stronger as the displacement of the initial conditions from the locus of equilibrium operating points increases.

However, only the dependence of the rate of variation of the scheduling variable on the input is really inherent to the nonlinear controller and for some choices of realisation the dependence on the initial conditions is weakened. Consider the situation when the non-zero rows of $\nabla_{\mathrm{x}} \rho$ are row eigenvectors of $\mathbf{A}$ in (6). Under these circumstances

$$
\dot{\rho}=\nabla_{x} \rho \mathbf{A x}+\nabla_{x} \rho \mathbf{B r}+\nabla_{x} \rho \mathbf{f}(\rho)+\nabla_{\mathrm{r}} \rho \dot{\mathbf{r}}=\Lambda \rho+\nabla_{\mathrm{x}} \rho \mathbf{f}(\rho)+\nabla_{\mathrm{x}} \rho \mathbf{B r}-\Lambda \nabla_{\mathrm{r}} \rho \mathbf{r}+\nabla_{\mathrm{r}} \rho \dot{\mathbf{r}}
$$

where $\Lambda$ is a diagonal matrix, the non-zero diagonal elements of which are eigenvalues of $\mathbf{A}$. A restriction on the rate of variation of the scheduling variable, $\rho$, imposes a restriction on the input, $\mathbf{r}$, and the initial conditions of the scheduling variable. There are no restrictions on those linear combinations of the state which are linearly independent of the scheduling variable.

Example Consider the non-linear controller

$$
\mathbf{x}=\mathbf{A x}+\mathbf{B r}+\mathbf{f}(\mathbf{x}, \mathbf{r}), \quad \mathrm{y}=\mathbf{C x}+\operatorname{Dr}+\mathbf{g}(\mathbf{x}, \mathbf{r})
$$

where $\mathbf{x}=\left[\begin{array}{ll}\mathrm{x}_{1} & \mathrm{x}_{2}\end{array}\right]^{\mathrm{T}}$ and $\rho=\mathrm{x}_{1}$ with

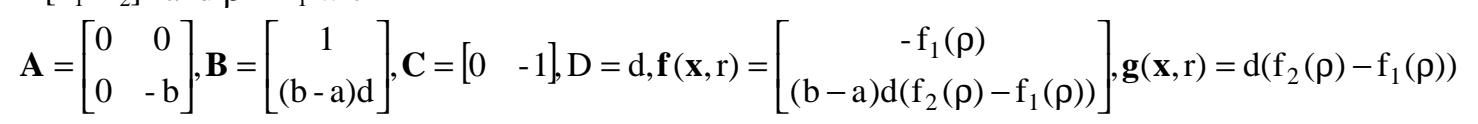

The transfer functions for the family of linear controllers, obtained by linearising the nonlinear controller, are 


$$
\frac{d(s+a)\left(s+\nabla_{\rho} f_{2}(\rho)\right)}{(s+b)\left(s+\nabla_{\rho} f_{1}(\rho)\right)}
$$

and the dynamic behaviour of the scheduling variable, $\rho$, is described by

$$
\dot{\rho}=-\mathrm{f}_{1}(\rho)+\mathrm{r}
$$

Of particular interest are those realisations, satisfying the extended local linear equivalence condition, for which the scheduling variable depends solely on the input; that is, for which $\nabla_{\mathrm{x}} \rho$ is zero. With these realisations, there are no restrictions on the initial conditions of the state: a restriction is imposed by the extent, to which the dynamics vary over the locus of equilibrium operating points, on the class of inputs only and not on the initial conditions. When the input is constant, the scheduling variable is constant and the dynamics of the controller are purely linear. The situation is exactly analogous to the exogenously gain-scheduled controller, (1).

\subsection{Equivalent families of linear controllers}

In section 3.1, realisations satisfying the extended local linear equivalence conditions are defined with reference to the form of the nonlinear controller. Equivalently, they can be defined with respect to the form of the family of linear controllers.

Associated with each family of linear controllers, (2), is the family of matrices

$$
\Sigma(\pi)=\left[\begin{array}{ll}
\mathbf{A}_{\pi} & \mathbf{B}_{\pi} \\
\mathbf{C}_{\pi} & \mathbf{D}_{\pi}
\end{array}\right]
$$

where the dimension of $\pi$ is q. Consider the difference matrix between a fixed member of the family, $\Sigma\left(\pi_{\mathbf{0}}\right)$, and any other member, $\Sigma\left(\pi_{1}\right)$.

$$
\Delta\left(\pi_{1}\right)=\Sigma\left(\pi_{\mathbf{0}}\right)-\left[\begin{array}{cc}
\mathbf{S}\left(\pi_{1}\right) & \mathbf{0} \\
\mathbf{0} & \mathbf{I}
\end{array}\right] \Sigma\left(\pi_{\mathbf{1}}\right)\left[\begin{array}{cc}
\mathbf{S}^{-1}\left(\pi_{1}\right) & \mathbf{0} \\
\mathbf{0} & \mathbf{I}
\end{array}\right]
$$

where $\mathbf{S}(\pi) \in \mathfrak{R}^{\mathrm{n} \times \mathrm{n}}$ is non-singular and corresponds to a state transformation with respect to which the dynamics of the linear controller at $\pi$ are unchanged. Provided that the rank of all $\Delta\left(\pi_{1}\right)$, for some $\mathbf{S}\left(\pi_{1}\right)$, is q and, in addition, the rows of all $\Delta\left(\pi_{1}\right)$ belong to a q-dimensional sub-space of $\Re^{(\mathrm{n}+\mathrm{p}) \times \mathrm{q}}$, then there exists a $\hat{\mathbf{f}}(\pi)$ and $\hat{\mathbf{g}}(\pi)$ such that

$$
\Delta\left(\pi_{1}\right) \equiv\left[\begin{array}{l}
\hat{\mathbf{f}}\left(\pi_{1}\right) \\
\hat{\mathbf{g}}\left(\pi_{1}\right)
\end{array}\right]\left[\begin{array}{ll}
\mathbf{M} & \mathbf{N}
\end{array}\right]
$$

where $[\mathbf{M ~} \mathbf{N}]$ is a constant matrix, has rank $\mathrm{q}$ and spans the q-dimensional sub-space. It follows that

$$
\Sigma(\pi) \equiv\left[\begin{array}{ll}
\mathbf{A}+\hat{\mathbf{f}}(\pi) \mathbf{M} & \mathbf{B}+\hat{\mathbf{f}}(\pi) \mathbf{N} \\
\mathbf{C}+\hat{\mathbf{g}}(\pi) \mathbf{M} & \mathbf{D}+\hat{\mathbf{g}}(\pi) \mathbf{N}
\end{array}\right]
$$

where

$$
\left[\begin{array}{ll}
\mathbf{A} & \mathbf{B} \\
\mathbf{C} & \mathbf{D}
\end{array}\right]=\Sigma\left(\boldsymbol{\pi}_{\mathrm{o}}\right)
$$

Assume $\pi$ is related to the q-dimensional scheduling variable, $\rho$, by the one-to-one mapping

$$
\pi=\mathbf{h}(\rho)
$$

and that there exist solutions, $\mathbf{f}(\rho)$ and $\mathbf{g}(\rho)$, of, respectively,

$$
\nabla_{\rho} \mathbf{f}(\rho)=\hat{\mathbf{f}}(\mathbf{h}(\rho)), \quad \nabla_{\rho} \mathbf{g}(\rho)=\hat{\mathbf{g}}(\mathbf{h}(\rho)),
$$

The latter assumption is satisfied when $\nabla_{\rho_{i}} \nabla_{\rho_{j}} \hat{\mathbf{f}}(\rho)=\nabla_{\rho_{j}} \nabla_{\rho_{i}} \dot{\mathbf{f}}(\rho), \nabla_{\rho_{i}} \nabla_{\rho_{j}} \hat{\mathbf{g}}(\rho)=\nabla_{\rho_{j}} \nabla_{\rho_{i}} \hat{\mathbf{g}}(\rho), \quad \forall i \neq j$, where $\rho_{\mathrm{i}}$ denotes the $\mathrm{i}^{\text {th }}$ element of $\rho$. When the family of controllers is derived by smoothly interpolating between a finite number of controller designs, this requirement is not restrictive. The usual gain-scheduling design approach is to design linear controllers for a number of distinct equilibrium operating points spanning the operating envelope. Moreover, the dimension the scheduling variable, $\rho$, is typically quite small. Hence, the condition, (32), is readily satisfied. The family of linear controllers, (2), can be realised by a nonlinear controller, (6), for which

$$
\rho=\mathbf{M x}+\mathbf{N r}
$$

provided that there exists, for all values of $\rho$, a solution of 


$$
\left[\begin{array}{ccc}
-\mathbf{A} & \mathbf{0} & -\mathbf{B} \\
-\mathbf{C} & \mathbf{I} & -\mathbf{D} \\
\mathbf{M} & \mathbf{0} & \mathbf{N}
\end{array}\right]\left[\begin{array}{l}
\mathbf{x}_{\mathbf{0}}(\rho) \\
\mathbf{y}_{\mathbf{0}}(\rho) \\
\mathbf{r}_{\mathbf{0}}(\rho)
\end{array}\right]=\left[\begin{array}{c}
\mathbf{f}(\rho) \\
\mathbf{g}(\rho) \\
\rho
\end{array}\right]
$$

such that $\left(\mathbf{r}_{0}\left(\rho_{1}\right), \mathbf{y}_{\mathbf{0}}\left(\rho_{1}\right)\right) \neq\left(\mathbf{r}_{\mathbf{0}}\left(\rho_{2}\right), \mathbf{y}_{\mathbf{0}}\left(\rho_{2}\right)\right)$ when $\rho_{1} \neq \rho_{2}$. Furthermore, the $\left(\mathbf{x}_{0}(\rho), \mathbf{y}_{\mathbf{0}}(\rho), \mathbf{r}_{\mathbf{0}}(\rho)\right)$ are the equilibrium operating points of the nonlinear controller.

It should be noted that the existence of a solution of (34) does not depend on the choice of $\mathbf{h}(\bullet)$ in (31). When the matrix on the left-hand side of (34) is full rank, the existence of a solution regardless of the choice of $\mathbf{h}(\bullet)$ is clear. When the matrix is not full rank, a solution of (34) exists provided, for some constant matrices $\mathbf{X}, \mathbf{Y}$ and $\mathbf{Z}$, dependent on $\mathbf{A}, \mathbf{B}, \mathbf{C}, \mathbf{D}, \mathbf{M}$ and $\mathbf{N}$ and not all zero,

$$
\mathbf{X f}(\rho)+\mathbf{Y g}(\rho)+\mathbf{Z} \rho=0
$$

for all $\rho$. The initial conditions for (32) must be chosen such that (35) is satisfied for some $\rho_{0}$; for example, choose $\rho_{\mathbf{0}}=0$ and the initial conditions for $(35)$ to be $\mathbf{f}(0)=\mathbf{g}(0)=0$. It follows that (35) is satisfied provided

$$
\mathbf{X} \nabla_{\rho} \mathbf{f}(\rho)+\mathbf{Y} \nabla_{\rho} \mathbf{g}(\rho)+\mathbf{Z}=0
$$

which is equivalent to

$$
\mathbf{X} \hat{\mathbf{f}}(\pi)+\mathbf{Y} \hat{\mathbf{g}}(\pi)+\mathbf{Z}=0
$$

Since (37) and the choice of the initial conditions for (35) are independent of $\mathbf{h}(\bullet)$, the existence of a solution of (34) does not depend on the choice of $\mathbf{h}(\bullet)$. Different $\mathbf{h}(\bullet)$ correspond to the different isomorphisms possible when defining the scheduling variable.

Clearly, not all families of linear controllers, (2), can be realised by a nonlinear controller, (6), satisfying the extended local linear equivalence condition. Hence, extended local linear equivalence might appear to be a rather strong condition.

\subsection{Utility of realisations}

Since extended local linear equivalence appears to be a rather strong condition, controllers satisfying it might be expected to be of limited utility. It is, therefore, necessary to determine whether requiring the gain-scheduled controller to be a member of the class of nonlinear controllers, which satisfy the extended local linear equivalence condition, is overly restrictive.

Consider a family of m-input single-output linear controllers. The transfer function relating the output, $\mathrm{y}(\mathrm{t})$, and the $\mathrm{i}^{\text {th }}$ input, $\mathrm{r}_{\mathrm{i}}(\mathrm{t})$, is

$$
Y(s)=d \frac{b_{1}^{i} s^{n}+b_{2}^{i} s^{n-1}+\ldots+b_{n}^{i} s+b_{n+1}^{i}}{s^{n}+a_{1} s^{n-1}+\ldots+a_{n-1} s+a_{n}} R_{i}(s)
$$

where $\mathrm{Y}(\mathrm{s})$ and $\mathrm{R}_{\mathrm{i}}(\mathrm{s})$ are, respectively, the Laplace transforms of $\mathrm{y}(\mathrm{t})$ and $\mathrm{r}_{\mathrm{i}}(\mathrm{t})$, and it is assumed that

$$
\mathrm{Y}(\mathrm{s}) / \mathrm{R}_{\mathrm{i}}(\mathrm{s}) \neq \mathrm{Y}(\mathrm{s}) / \mathrm{R}_{\mathrm{j}}(\mathrm{s}) \quad \forall \mathrm{i} \neq \mathrm{j}
$$

The coefficient, $d$, is superfluous and could be absorbed into the coefficients, $b_{j}{ }_{j}^{i}$, without loss of generality. Accordingly, assuming $d$ is unity, it is evident that each transfer function is completely specified by its $n+1$ numerator coefficients, $b_{j}{ }_{j}$, and $n$ denominator coefficients, $a_{i}$. Since the $m$ transfer functions have, without loss of generality, a common denominator, the input-output characteristics of the $\mathrm{n}^{\text {th }}$ order $\mathrm{m}$-input single-output controllers are specified by $\mathrm{n}+\mathrm{m}(\mathrm{n}+1)$ coefficients in total. A corresponding minimal state-space representation is

$$
\begin{aligned}
& {\left[\begin{array}{c}
\dot{\mathrm{x}}_{1} \\
\dot{\mathrm{x}}_{2} \\
\vdots \\
\dot{\mathrm{x}}_{\mathrm{n}-1} \\
\dot{\mathrm{x}}_{\mathrm{n}}
\end{array}\right]=\left[\begin{array}{cccccc}
-\mathrm{a}_{1} & 1 & 0 & \cdots & 0 & 0 \\
-\mathrm{a}_{2} & 0 & 1 & & 0 & 0 \\
\vdots & \vdots & \vdots & & \vdots & \vdots \\
-\mathrm{a}_{\mathrm{n}-1} & 0 & 0 & & 0 & 1 \\
-\mathrm{a}_{\mathrm{n}} & 0 & 0 & \cdots & 0 & 0
\end{array}\right]\left[\begin{array}{c}
\mathrm{x}_{1} \\
\mathrm{x}_{2} \\
\vdots \\
\mathrm{x}_{\mathrm{n}-1} \\
\mathrm{x}_{\mathrm{n}}
\end{array}\right]+\left[\begin{array}{cccc}
-\mathrm{a}_{1} \mathrm{~b}_{1}^{1}+\mathrm{b}_{2}^{1} & -\mathrm{a}_{1} \mathrm{~b}_{1}^{2}+\mathrm{b}_{2}^{2} & \cdots & -\mathrm{a}_{1} \mathrm{~b}_{1}^{\mathrm{m}}+\mathrm{b}_{2}^{\mathrm{m}} \\
-\mathrm{a}_{2} \mathrm{~b}_{1}^{1}+\mathrm{b}_{3}^{1} & -\mathrm{a}_{2} \mathrm{~b}_{1}^{2}+\mathrm{b}_{3}^{1} & & -\mathrm{a}_{2} \mathrm{~b}_{1}^{\mathrm{m}}+\mathrm{b}_{3}^{\mathrm{m}} \\
\vdots & \vdots & \vdots \\
\mathrm{a}_{\mathrm{n}-1} \mathrm{~b}_{1}^{1}+\mathrm{b}_{\mathrm{n}}^{1} & -\mathrm{a}_{\mathrm{n}-1} \mathrm{~b}_{1}^{2}+\mathrm{b}_{\mathrm{n}}^{2} & & -\mathrm{a}_{\mathrm{n}-1} \mathrm{~b}_{1}^{\mathrm{m}}+\mathrm{b}_{\mathrm{n}}^{\mathrm{m}} \\
-\mathrm{a}_{\mathrm{n}} \mathrm{b}_{1}^{1}+\mathrm{b}_{\mathrm{n}+1}^{1} & -\mathrm{a}_{\mathrm{n}} \mathrm{b}_{1}^{2}+\mathrm{b}_{\mathrm{n}+1}^{2} & \cdots & -\mathrm{a}_{\mathrm{n}} \mathrm{b}_{1}^{\mathrm{m}}+\mathrm{b}_{\mathrm{n}+1}^{\mathrm{m}}
\end{array}\right]\left[\begin{array}{c}
\mathrm{r}_{1} \\
\mathrm{r}_{2} \\
\vdots \\
r_{\mathrm{m}}
\end{array}\right]} \\
& \mathrm{y}=\mathrm{d}\left[\begin{array}{lllll}
1 & 0 & \cdots & 0 & 0
\end{array}\right]\left[\begin{array}{c}
\mathrm{x}_{1} \\
\mathrm{x}_{2} \\
\vdots \\
\mathrm{x}_{\mathrm{n}-1} \\
\mathrm{x}_{\mathrm{n}}
\end{array}\right]+\mathrm{d}\left[\begin{array}{llll}
\mathrm{b}_{1}^{1} & \mathrm{~b}_{1}^{2} & \cdots & \mathrm{b}_{1}^{\mathrm{m}} \\
\mathrm{r}_{2} \\
\vdots \\
\mathrm{r}_{\mathrm{m}}
\end{array}\right]
\end{aligned}
$$


where, owing to the particular choice of states, the transfer function coefficients appear in a straightforward manner in the state-space matrices. Of course any other, minimal, state-space representation satisfying the input-output relationships, (38), may be derived from (39) by a suitable state transformation.

It follows from (7) that a family of linear controllers corresponding to a nonlinear controller satisfying the extended local linear equivalence condition has, at most, $q(n+1)$ degrees of freedom. In all but the exceptional case when $q=m+1$ (note, $\mathrm{q} \leq \mathrm{m}+1), \mathrm{q}(\mathrm{n}+1)<\mathrm{n}+\mathrm{m}(\mathrm{n}+1)$ and not all of the coefficients of (38) can be freely assigned; that is, only a subset of $q(n+1)$ coefficients in (38) can be scheduled independently (subject to satisfying (32)), with the remaining coefficients either dependent on this subset or constant. The identity of the constant coefficients, and the relationships between the remainder, depend on the definition, in terms of linear combinations of the elements of the state and input, of $\rho$. Nevertheless, with few exceptions, all gain-scheduling requirements can be accommodated since, with the penalty of a non-minimal realisation, the order, $n$, of the controller may be increased to provide an arbitrary degree of flexibility. A family of linear controllers, equivalent to the nonlinear controller satisfying the extended local linear equivalence condition, may be constructed to approximate closely a family of previously designed, perhaps lower order, linear controllers. Alternatively, during synthesis, the linear controllers can be directly designed to have the required pattern of coefficient scheduling dependence.

Example The family of linear controllers, designed for a wind turbine in Leith \& Leithead (1996), consist of a fixed component

$$
\mathrm{K}(\mathrm{s})=\mathrm{C}(\mathrm{s}) / \mathrm{s}
$$

where

$$
\mathrm{C}(\mathrm{s})=\frac{(\mathrm{s}+1.7)(\mathrm{s}+1.8)\left(\mathrm{s}^{2}+7.59 \mathrm{~s}+68.06\right)\left(\mathrm{s}^{2}+2 \mathrm{~s}+104.04\right)\left(\mathrm{s}^{2}+3 \mathrm{~s}+416.16\right) 2209}{(\mathrm{~s}+0.3)(\mathrm{s}+3.7)(\mathrm{s}+30)(\mathrm{s}+100)\left(\mathrm{s}^{2}+11 \mathrm{~s}+104.04\right)\left(\mathrm{s}^{2}+8 \mathrm{~s}+416.16\right)\left(\mathrm{s}^{2}+65.8 \mathrm{~s}+2209\right)}
$$

together with a component, which varies with the equilibrium operating point,.

$$
\mathrm{C}_{\pi}(\mathrm{s})=\mathrm{g}(\pi) \frac{0.00147 \mathrm{~s}^{2}+0.111 \mathrm{~s}+1}{\mathrm{~s}^{2}+\mathrm{a}_{1}(\pi) \mathrm{s}+\mathrm{a}_{2}(\pi)}
$$

where $\pi$ parameterises the equilibrium operating points. The choice of $\pi$ is, of course, not unique. Selecting $\pi$ to be related to the wind speed, $\mathrm{V}$, by

$$
\pi=-0.044 \mathrm{~V}^{2}+2.95 \mathrm{~V}-24.97
$$

the value of $\pi$ corresponds to the pitch angle of the turbine rotor blades, at the equilibrium operating points, and

$$
\mathrm{a}_{1}(\pi)=-0.033 \pi^{2}+0.750 \pi+3.375, \mathrm{a}_{2}(\pi)=2.600 \pi+58.040, \mathrm{~g}(\pi)=0.138 \pi+0.298
$$

In (40c) all the poles vary with the operating point while the zeroes are constant. Suppose it is required, instead, that the zeroes vary while the poles are constant. The family of linear controllers, (40), can be re-designed such that

$$
\mathrm{K}^{\prime}(\mathrm{s})=\mathrm{C}^{\prime}(\mathrm{s}) / \mathrm{s}
$$

where

$$
\mathrm{C}^{\prime}(\mathrm{s})=\frac{(\mathrm{s}+1.7)(\mathrm{s}+1.8)\left(\mathrm{s}^{2}+2 \mathrm{~s}+104.04\right)\left(\mathrm{s}^{2}+3 \mathrm{~s}+416.16\right) 2209}{(\mathrm{~s}+0.3)(\mathrm{s}+3.7)\left(\mathrm{s}^{2}+11 \mathrm{~s}+104.04\right)\left(\mathrm{s}^{2}+8 \mathrm{~s}+416.16\right)\left(\mathrm{s}^{2}+65.8 \mathrm{~s}+2209\right)}
$$

and

$$
C^{\prime}{ }_{\pi}(s)=\frac{b_{1}\left(\pi^{\prime}\right) s^{4}+b_{2}\left(\pi^{\prime}\right) s^{3}+b_{3}\left(\pi^{\prime}\right) s^{2}+b_{4}\left(\pi^{\prime}\right) s+b_{5}\left(\pi^{\prime}\right)}{s^{4}+135.8 s^{3}+3818 s^{2}+26150 s+204000}
$$

Selecting $\pi^{\prime}$ to be related to the wind speed, $\mathrm{V}$, by

$$
\pi^{\prime}=-0.0027 \mathrm{~V}^{4}+0.37 \mathrm{~V}^{3}-19.31 \mathrm{~V}^{2}+469.45 \mathrm{~V}-3404.7=-1.42 \pi^{2}+88.51 \pi-311.72
$$

the value of $\pi^{\prime}$ corresponds to the input to $\mathrm{C}^{\prime}(\mathrm{s})$, at the equilibrium operating points, and

$\mathrm{b}_{1}\left(\pi^{\prime}\right)=4.82 \times 10^{-6} \pi^{\prime 2}-1.15 \times 10^{-3} \pi^{\prime}+1.042, \mathrm{~b}_{2}\left(\pi^{\prime}\right)=3.34 \times 10^{-5} \pi^{\prime 2}+1.68 \times 10^{-3} \pi^{\prime}+13.57$,

$b_{3}\left(\pi^{\prime}\right)=2.78 \times 10^{-4} \pi^{\prime 2}+3.15 \times 10^{-2} \pi^{\prime}+146.33, b_{4}\left(\pi^{\prime}\right)=5.82 \times 10^{-4} \pi^{\prime 2}+0.37 \pi^{\prime}+676.16$,

$\mathrm{b}_{5}\left(\pi^{\prime}\right)=1.38 \times 10^{-3} \pi^{\prime 2}+1.93 \pi^{\prime}+2295.00$

The required scheduling of transfer function coefficients has been achieved. As might be expected, in comparison to the order of $\mathrm{C}_{\pi}(\mathrm{s})$, the order of $\mathrm{C}^{\prime}{ }_{\pi}(\mathrm{s})$ is increased from 2 to 4 . However, the overall order of the controller is not increased since, in comparison to the order of $\mathrm{C}(\mathrm{s})$, the order of $\mathrm{C}^{\prime}(\mathrm{s})$ is decreased from 10 to 8 . The Bode plots of the two families of linear controllers, $C_{\pi}(s) C(s)$ and $C^{\prime}(s) C^{\prime}(s)$, are effectively indistinguishable at equivalent equilibrium operating points.

Although the normal motivation, in this context, for converting a family of linear controllers from one form to another would be to change the family from one not satisfying the extended local linear equivalence condition to one satisfying this condition, on this occasion both $\mathrm{C}_{\pi}(\mathrm{s})$, with the scheduling variable its output, and $\mathrm{C}_{\pi}^{\prime}(\mathrm{s})$, with the scheduling variable its input, are equivalent to nonlinear controllers satisfying the extended local linear equivalence condition. This example, thereby, also illustrates the dependence, on the definition of the scheduling variable, of the 
manner in which the linear controllers' transfer function coefficients are related to the scheduling variable; that is, the identity of the constant coefficients and the interdependence of the remainder.

The above arguments can clearly be extended to the MIMO case. Hence, in general, any restriction on the design of gain-scheduled controllers, due to adopting a realisation satisfying the extended local linear equivalence condition, can be expected to be rather weak. The apparent strength of the extended local linear equivalence condition is deceptive, albeit, at the expense of adopting realisations of, perhaps, non-minimal degree.

\section{Practical realisations}

In section 3, the properties of particular nonlinear controllers, namely those satisfying the extended local linear equivalence condition, are investigated. They are minimally nonlinear but sufficiently flexible to act as realisations for all gain-scheduled families of linear controllers. However, several issues central to the practical design and realisation of gain-scheduled controllers are not addressed: firstly, designing the family of linear controllers, for which the coefficients of the transfer functions are not independent of each other but must satisfy certain relationships; secondly, determining the nonlinear functions of the scheduling variable in the nonlinear controller, the partial derivatives of which are related to the linear controllers' transfer function coefficients; thirdly, ensuring that the controller input and output have appropriate values at the equilibrium operating points. These issues are discussed below.

\subsection{Separation of coefficients}

The first issue, namely the interdependence of the linear controllers' transfer function coefficients, is resolved by separating a subset of the coefficients, all of which may be essentially freely scheduled, with the remaining coefficients constant. Although all of the previously considered examples, having been chosen for simplicity and clarity, do conform to this resolution of the interdependence of the linear controllers' transfer function coefficients, it is clear from (7) that, in general, the family of linear controllers need not. Whether the separation of the coefficients occurs is dependent on the definition of the scheduling variable. It is sufficient to investigate $\mathrm{m}$-input single-output controllers since a general MIMO controller may be obtained by suitably combining several multiple-input single-output controllers.

Consider the m-input single-output nonlinear controller realisation, depicted in figure 4

$$
\left[\begin{array}{c}
\dot{\mathrm{x}}_{1} \\
\dot{\mathrm{x}}_{2} \\
\vdots \\
\dot{\mathrm{x}}_{\mathrm{n}-1} \\
\dot{\mathrm{x}}_{\mathrm{n}}
\end{array}\right]=\left[\begin{array}{cccccc}
-\mathrm{a}_{1} & 1 & 0 & \cdots & 0 & 0 \\
-\mathrm{a}_{2} & 0 & 1 & & 0 & 0 \\
\vdots & \vdots & \vdots & & \vdots & \vdots \\
-\mathrm{a}_{\mathrm{n}-1} & 0 & 0 & & 0 & 1 \\
-\mathrm{a}_{\mathrm{n}} & 0 & 0 & \cdots & 0 & 0
\end{array}\right]\left[\begin{array}{c}
\mathrm{x}_{1} \\
\mathrm{x}_{2} \\
\vdots \\
\mathrm{x}_{\mathrm{n}-1} \\
\mathrm{x}_{\mathrm{n}}
\end{array}\right]+\left[\begin{array}{c}
-\mathrm{a}_{1} \mathrm{~B}_{1}(\mathbf{r})+\mathrm{B}_{2}(\mathbf{r}) \\
-\mathrm{a}_{2} \mathrm{~B}_{1}(\mathbf{r})+\mathrm{B}_{3}(\mathbf{r}) \\
\vdots \\
-\mathrm{a}_{\mathrm{n}-1} \mathrm{~B}_{1}(\mathbf{r})+\mathrm{B}_{\mathrm{n}}(\mathbf{r}) \\
-\mathrm{a}_{\mathrm{n}} \mathrm{B}_{1}(\mathbf{r})+\mathrm{B}_{\mathrm{n}+1}(\mathbf{r})
\end{array}\right], \mathrm{y}=\left[\begin{array}{lllll}
1 & 0 & \cdots & 0 & 0
\end{array}\right]\left[\begin{array}{c}
\mathrm{x}_{1} \\
\mathrm{x}_{2} \\
\vdots \\
\mathrm{x}_{\mathrm{n}-1} \\
\mathrm{x}_{\mathrm{n}}
\end{array}\right]+\mathrm{B}_{1}(\mathbf{r})
$$

for some functions $B_{j}(\bullet), j=1,2, . . n+1$, where $\mathbf{r}=\left[\begin{array}{llll}r_{1} & r_{2} & \cdots & r_{m}\end{array}\right]^{T}$. Provided that the locus of equilibrium operating points can be parameterised by the input, $\mathbf{r}$, the controller, (42), has the form required by (6). Hence, this controller realisation satisfies the extended local linear equivalence condition with the scheduling variable simply the input, $\mathbf{r}$. Moreover, at any equilibrium operating point, the linearisation of (42) and its transfer functions have the forms, (39) and (38), respectively, with

$$
\mathrm{b}_{\mathrm{j}}^{\mathrm{i}}=\nabla_{\mathrm{r}_{\mathrm{i}}} \mathrm{B}_{\mathrm{j}}\left(\mathbf{r}_{\mathrm{o}}\right), \quad \mathrm{i}=1,2, . ., \mathrm{m}, \quad \mathrm{j}=1,2, . ., \mathrm{n}+1
$$

Adoption of the controller realisation of figure 4 enables the extended local linear equivalence condition to be satisfied for families of m-input single-output linear controllers, for which the controller zeroes are scheduled essentially independently with respect to the input, $\mathbf{r}$, but the controller poles are fixed. Hence, the requirement to separate a subset of coefficients, those of the numerators of the transfer functions in this realisation, all of which can be freely scheduled, with the remaining coefficients, those of the denominator, constant, is met. It should be noted that the linear controllers associated with (42) have the maximum possible scheduling degrees of freedom, $(n+1) q$.

Alternatively, consider the $\mathrm{m}$-input single-output nonlinear controller realisation, depicted in figure 5 ,

$$
\left[\begin{array}{c}
\dot{\mathrm{x}}_{1} \\
\dot{\mathrm{x}}_{2} \\
\vdots \\
\dot{\mathrm{x}}_{\mathrm{n}-1} \\
\dot{\mathrm{x}}_{\mathrm{n}}
\end{array}\right]=\left[\begin{array}{cccccc}
0 & 1 & 0 & \cdots & 0 & 0 \\
0 & 0 & 1 & & 0 & 0 \\
\vdots & \vdots & \vdots & & \vdots & \vdots \\
0 & 0 & 0 & & 0 & 1 \\
0 & 0 & 0 & \cdots & 0 & 0
\end{array}\right]\left[\begin{array}{c}
\mathrm{x}_{1} \\
\mathrm{x}_{2} \\
\vdots \\
\mathrm{x}_{\mathrm{n}-1} \\
\mathrm{x}_{\mathrm{n}}
\end{array}\right]+\left[\begin{array}{c}
\mathrm{B}_{2}(\mathrm{z}, \hat{\mathbf{r}})+\mathrm{b}_{2}^{1} \mathrm{r}_{1} \\
\mathrm{~B}_{3}(\mathrm{z}, \hat{\mathbf{r}})+\mathrm{b}_{3}^{1} \mathrm{r}_{1} \\
\vdots \\
\mathrm{B}_{\mathrm{n}}(\mathrm{z}, \hat{\mathbf{r}})+\mathrm{b}_{\mathrm{n}}^{1} \mathrm{r}_{1} \\
\mathrm{~B}_{\mathrm{n}+1}(\mathrm{z}, \hat{\mathbf{r}})+\mathrm{b}_{\mathrm{n}+1}^{1} \mathrm{r}_{1}
\end{array}\right], \mathrm{y}=\mathrm{G}(\mathrm{z})
$$


for some functions $\mathrm{G}(\bullet), \mathrm{B}_{1}(\bullet)$ and $\mathrm{B}_{\mathrm{j}}(\bullet, \bullet), \mathrm{j}=2,3 \ldots \mathrm{n}+1$, where, $\hat{\mathbf{r}}=\left[\begin{array}{llll}\mathrm{r}_{2} & \mathrm{r}_{3} & \cdots & \mathrm{r}_{\mathrm{m}}\end{array}\right]^{\mathrm{T}}$ and $\mathrm{z}=\mathrm{x}_{1}+\mathrm{b}_{1}^{1} \mathrm{r}_{1}+\mathrm{B}_{1}(\hat{\mathbf{r}})$. Provided that the locus of equilibrium operating points can be parameterised by input, $\hat{\mathbf{r}}$, together with the output, $\mathrm{y}$, then the controller, (44), has the form required by (6). Hence, this controller realisation satisfies the extended local linear equivalence condition with the scheduling variable $\left(\mathrm{x}_{1}+\mathrm{b}_{1}^{1} \mathrm{r}_{1}, \hat{\mathbf{r}}^{\mathrm{T}}\right)^{\mathrm{T}}$. Moreover, at any equilibrium operating point, the linearisation of (44) and its transfer functions have the forms (39) and (38), respectively, with

$$
\begin{aligned}
& \mathrm{a}_{\mathrm{j}}=-\nabla_{\mathrm{z}} \mathrm{B}_{\mathrm{j}+1}\left(\mathrm{z}_{\mathrm{o}}, \hat{\mathbf{r}}_{\mathrm{o}}\right), \mathrm{j}=1,2, . . \mathrm{n}, \quad \mathrm{d}=\nabla_{\mathrm{z}} \mathrm{G}\left(\mathrm{z}_{\mathrm{o}}\right) ; \\
& \mathrm{b}_{1}{ }^{\mathrm{i}}=\nabla_{\mathrm{r}_{\mathrm{i}}} \mathrm{B}_{1}\left(\hat{\mathbf{r}}_{\mathrm{o}}\right), \mathrm{i}=2,3, . . \mathrm{m} ; \quad \mathrm{b}_{\mathrm{j}}{ }^{\mathrm{i}}=\nabla_{\mathrm{r}_{\mathrm{i}}} \mathrm{B}_{\mathrm{j}}\left(\mathrm{z}_{\mathrm{o}}, \hat{\mathbf{r}}_{\mathrm{o}}\right), \quad \mathrm{i}=2,3, . . \mathrm{m}, \mathrm{j}=2, . . \mathrm{n}+1
\end{aligned}
$$

Without loss of generality, it may be assumed that the derivative of $\mathrm{G}(\bullet)$ is either strictly positive or strictly negative. It follows that $\mathrm{G}(\bullet)$ is monotonic and $\mathrm{G}^{-1}(\bullet)$ exists. Hence, the nonlinear controller, (44), may be interpreted as being scheduled on the output $y$, albeit implicitly, and the elements of the input, $\mathrm{r}_{2}, \ldots \mathrm{r}_{\mathrm{m}}$.

Adoption of the controller realisation of figure 5 enables the extended local linear equivalence condition to be satisfied for families of m-input single-output linear controllers, for which the controller poles are scheduled essentially independently with respect to the output, $y$, and the elements of the input, $r_{2}, \ldots r_{m}$. In addition, other than the transfer function relating $\mathrm{r}_{1}$ to $\mathrm{y}$, for which the zeroes are fixed, the zeroes of the transfer functions relating the inputs individually to the output are also scheduled essentially independently with respect to the output, $y$, and the elements of the input, $\mathrm{r}_{2}, \ldots \mathrm{r}_{\mathrm{m}}$. Hence, the requirement to separate a subset of coefficients, all of which can be essentially freely scheduled with the remaining coefficients constant, is met for this realisation, with the subset consisting of all the transfer function coefficients except those of the numerator of the transfer function relating $\mathrm{r}_{1}$ to $\mathrm{y}$. The linear controllers associated with (44) have the maximum possible scheduling degrees of freedom, $(\mathrm{n}+1) \mathrm{q}$. However, only nq coefficients are scheduled with respect to both $y$ and, $r_{2}, \ldots r_{m}$ with (q-1) coefficients, namely $b_{1}{ }^{i}, i=2, \ldots$ m, scheduled with respect to $r_{2}, \ldots r_{m}$ alone and one coefficient, namely $d$, scheduled with respect to $y$ alone.

The realisation, (44), is equivalent, in a SISO context, to the realisation satisfying the extended local linear equivalence condition described in Leith \& Leithead (1996).

Example With reference to the wind turbine controllers described in the Example in section 3.5, the transfer function numerator coefficients of the family of linear controllers, $(41 \mathrm{~b})$, are scheduled whilst the denominator coefficients are constant. It is appropriate, therefore, to identify the controllers, (41b) with the realisation, (42), specialised to a SISO system. In other words, the scheduling variable can be identified as the input to the nonlinear controller equivalent to the $\mathrm{C}_{\pi}^{\prime}(\mathrm{s})$, namely, $\mathrm{r}$, and the nonlinear controller, denoted $\mathrm{C}_{\pi}^{\prime}$, satisfying the extended local linear equivalence condition, is

$$
\left[\begin{array}{l}
\dot{x}_{1} \\
\dot{x}_{2} \\
\dot{x}_{3} \\
\dot{x}_{4}
\end{array}\right]=\left[\begin{array}{cccc}
-135.8 & 1 & 0 & 0 \\
-3818 & 0 & 1 & 0 \\
-26150 & 0 & 0 & 1 \\
-204000 & 0 & 0 & 0
\end{array}\right]\left[\begin{array}{l}
x_{1} \\
x_{2} \\
x_{3} \\
x_{4}
\end{array}\right]+\left[\begin{array}{c}
-135.8 B_{1}(r)+B_{2}(r) \\
-3818 B_{1}(r)+B_{3}(r) \\
-26150 B_{1}(r)+B_{4}(r) \\
-204000 B_{1}(r)+B_{5}(r)
\end{array}\right], \quad y=x_{1}+B_{1}(r)
$$

where

$$
\begin{aligned}
& \mathrm{B}_{1}(\mathrm{r})=1.60 \times 10^{-6} \mathrm{r}^{3}-5.75 \times 10^{-4} \mathrm{r}^{2}+1.042 \mathrm{r}, \mathrm{B}_{2}(\mathrm{r})=1.11 \times 10^{-5} \mathrm{r}^{3}+8.40 \times 10^{-4} \mathrm{r}^{2}+13.57 \mathrm{r}, \\
& \mathrm{B}_{3}(\mathrm{r})=9.27 \times 10^{-5} \mathrm{r}^{3}+1.57 \times 10^{-2} \mathrm{r}^{2}+146.33 \mathrm{r}, \mathrm{B}_{4}(\mathrm{r})=1.94 \times 10^{-4} \mathrm{r}^{3}+0.18 \mathrm{r}^{2}+676.16 \mathrm{r}, \\
& \mathrm{B}_{5}(\mathrm{r})=4.6 \times 10^{-4} \mathrm{r}^{3}+0.96 \mathrm{r}^{2}+2295.00 \mathrm{r}
\end{aligned}
$$

On the other hand, the transfer function denominator coefficients, together with the overall gain, of the family of linear controllers, (40b), are scheduled whilst the numerator coefficients are constant. It is appropriate, therefore, to identify the controller, (40b) with the realisation, (44), specialised to a SISO system. In other words, the scheduling variable can be identified as the output from the nonlinear controller equivalent to the $\mathrm{C}_{\pi}(\mathrm{s})$, namely $\mathrm{y}$, and the nonlinear controller, denoted $\mathrm{C}_{\pi}$, satisfying the extended local linear equivalence condition, is

$$
\left[\begin{array}{l}
\dot{\mathrm{x}}_{1} \\
\dot{\mathrm{x}}_{2}
\end{array}\right]=\left[\begin{array}{ll}
0 & 1 \\
0 & 0
\end{array}\right]\left[\begin{array}{l}
\mathrm{x}_{1} \\
\mathrm{x}_{2}
\end{array}\right]+\left[\begin{array}{c}
-\mathrm{B}_{2}(\mathrm{z})+0.111 \mathrm{r} \\
-\mathrm{B}_{3}(\mathrm{z})+\mathrm{r}
\end{array}\right], \quad \mathrm{y}=\mathrm{G}(\mathrm{z})
$$

where

$$
\begin{aligned}
& \mathrm{z}=\mathrm{x}_{1}+0.00147 \mathrm{r}, \quad \mathrm{G}(\mathrm{z})=2.159\left(\mathrm{e}^{0.138 \mathrm{z}}-1\right) \\
& \mathrm{B}_{2}(\mathrm{z})=-0.557 \mathrm{e}^{0.276 \mathrm{z}}+13.96 \mathrm{e}^{0.138 \mathrm{z}}+1.602 \mathrm{z}, \quad \mathrm{B}_{3}(\mathrm{z})=40.67 \mathrm{e}^{0.138 \mathrm{z}}+52.42 \mathrm{z}
\end{aligned}
$$

Notice that whilst the transfer function coefficients of the linear family, (40b), are parameterised by the controller output, the associated nonlinear functions employed in the realisation, (47), are expressed in terms of $\mathrm{z}$. The realisation is explicitly scheduled with respect to $\mathrm{z}$ but, since $\mathrm{G}(\mathrm{z})$ is invertible, implicitly scheduled with respect to the output, y.

The dependence of the form of the family of linear controllers on the definition of the scheduling variable is very evident in the foregoing. It is not difficult to devise other realisations, corresponding to different definitions of the scheduling variable, which achieve the separation of scheduling dependent coefficients in other ways; for example, the 
Example of section 3.3. When, as in section 3.4, the members of the family of linear controllers are defined in general state-space form, their equivalence to a nonlinear controller satisfying the extended local linear equivalence condition is not obvious. In contrast, when, as above, the members are defined in transfer function form or, equivalently, in a canonical state-space form which is explicitly parameterised by the transfer function coefficients, their equivalence is obvious due to the strong correspondence between the forms of the nonlinear controller and the members of the family of linear controllers. In particular, their equivalence and the appropriate definition of the scheduling variable are easily identified for those families exhibiting separation of the scheduling dependent coefficients. This ease of identification greatly assists the design of linear controllers to be realised as a gain-scheduled nonlinear controller satisfying the extended local linear equivalence condition for a particular definition of the scheduling variable. Conversely, it is clear that the realisation of the gain-scheduled controller can be facilitated by the judicious choice of an appropriate scheduling variable.

In general, there are many linear combinations of the elements of $\left(\mathbf{x}_{\mathbf{0}}, \mathbf{y}_{\mathbf{0}}, \mathbf{r}_{\mathbf{0}}\right)$, which parameterise the equilibrium operating points; that is, there are many possible choices of scheduling variable. Choosing the scheduling variable, as above, to facilitate the realisation of the nonlinear gain-scheduled controller might appear to conflict with the more usual approach of choosing the scheduling variable on the basis of physical insight whereby some combinations of the elements of the input, state and output, representing the variables with respect to which the plant dynamics or the control objectives (see Leith \& Leithead 1996) vary, are chosen. For the wind turbine of the Example in section 3.5, the controller would ideally be scheduled with respect to the wind speed. Unfortunately, a measurement of the wind speed experienced by the turbine is impossible (Leith \& Leithead 1996). However, provided the controller is effective, the pitch angle of the rotor blades acts as a good estimate of the wind speed. Moreover, since the bandwidth of the blade pitch actuator is, typically, large in comparison to the bandwidth of the closed-loop controlled system, the pitch demand to the actuator, i.e. the controller output, also acts as a good estimate of the wind speed. Accordingly, a suitable controller for the wind turbine is $\mathrm{K}(\mathrm{s}) \mathrm{C}_{\pi}$, with $\mathrm{K}(\mathrm{s})$ positioned before $\mathrm{C}_{\pi}$, and $\mathrm{C}_{\pi}$ realised as (47) so that the scheduling variable is the output of the controller. Nevertheless, it is only the rate of variation of scheduling that really matters and any scheduling variable, the spectra of which has similar bandwidth, would be equally suitable. For the wind turbine, by design, the input and output of the $\mathrm{C}_{\pi}^{\prime}(\mathrm{s})$ have similar bandwidths. Hence, an equally suitable controller is $\mathrm{K}^{\prime}(\mathrm{s}) \mathrm{C}^{\prime}{ }_{\pi}$, with $\mathrm{K}^{\prime}(\mathrm{s})$ positioned before $\mathrm{C}^{\prime}{ }_{\pi}$, and $\mathrm{C}^{\prime}{ }_{\pi}$ realised as (46). Consequently, there is no conflict between choosing the scheduling variable to facilitate the realisation of the nonlinear gain-scheduling controller and choosing it on the basis of physical insight.

\subsubsection{Utility revisited}

Taking into account the number of transfer function coefficients that can be freely scheduled (namely, $m(n+1)$ out of $\mathrm{n}+\mathrm{m}(\mathrm{n}+1)$ for the realisations investigated above), the range of possible choices of the scheduling variable (equivalently, the forms of the family of linear controllers) and the ability to design/re-design linear controllers to have a specific form (see Example in section 3.5), then, even when restricted to those achieving separation of the scheduling dependent coefficients, the flexibility of realisations satisfying the extended local linear equivalence condition is apparent. Clearly, it is possible to realise, with few exceptions, all gain-scheduled controllers as nonlinear controllers satisfying the extended local linear equivalence condition and requiring the controller to be so realised is not at all restrictive.

\subsection{Velocity-based realisations}

The second issue, namely the requirement to determine the nonlinear functions of the scheduling variable in the nonlinear controller, is resolved by adopting realisations for which linear controllers are obtained by "freezing" the scheduling variable at its current value. Implicitly, this procedure requires the nonlinear controller to be linearised at any operating point, for which the value of the scheduling variable is within its domain, and not just at the equilibrium operating points. However, this is possible for nonlinear controllers satisfying the extended local linear equivalence condition as discussed in section 3.1.2.

The nonlinear controller, (6), can be reformulated, by differentiating, as

$$
\begin{aligned}
& \dot{\mathbf{w}}=\left(\mathbf{A}+\nabla_{\rho} \mathbf{f}(\rho) \nabla_{x} \rho\right) \mathbf{w}+\left(\mathbf{B}+\nabla_{\rho} \mathbf{f}(\rho) \nabla_{\mathbf{r}} \rho\right) \dot{\mathbf{r}} \\
& \dot{\mathbf{y}}=\left(\mathbf{C}+\nabla_{\rho} \mathbf{g}(\rho) \nabla_{x} \rho\right) \mathbf{w}+\left(\mathbf{D}+\nabla_{\rho} \mathbf{g}(\rho) \nabla_{\mathbf{r}} \rho\right) \dot{\mathbf{r}} \\
& \dot{\rho}=\nabla_{x} \rho \mathbf{w}+\nabla_{\mathbf{r}} \rho \dot{\mathbf{r}}
\end{aligned}
$$

where $\mathbf{w}=\dot{\mathbf{x}}$. Dynamically, (48), with appropriate initial conditions, namely,

$$
\mathbf{w}(0)=\mathbf{A x}(0)+\mathbf{B r}(0)+\mathbf{f}(\rho(0)), \quad \rho(\mathbf{0})=\nabla_{\mathbf{x}} \rho \mathbf{x}(0)+\nabla_{\mathbf{r}} \rho \mathbf{r}(0)
$$

and (6) are equivalent. Indeed, when

$$
\begin{aligned}
& \mathbf{w}=\mathbf{A x}+\mathbf{B r}+\mathbf{f}(\rho) \\
& \mathbf{y}=\mathbf{C} \mathbf{x}+\mathbf{D r}+\mathbf{g}(\rho)
\end{aligned}
$$


is invertible such that $\mathbf{x}$ may be expressed as a function of $\mathbf{w}, \mathbf{r}$ and $\mathbf{y}$, this reformulation is equivalent to an algebraic state transformation ( $(48 \mathrm{c})$ can then be replaced by a direct evaluation of $\rho$ ). Provided that $\mathbf{r}$, required as the input, and perhaps $\mathbf{r}$, when required by the scheduling variable, are available and provided that any unwanted constant displacement of the state or output, due to the differentiation of the input followed by the integration to determine the output from $\dot{\mathbf{y}}$, is avoided, then (48) is a viable alternative representation for (6). Comparing the linear controller, obtained by setting $\rho$ to $\rho_{\mathbf{o}}$ in (48), to the linear controller, (7), it is evident that the transfer functions relating $\mathbf{y}$ to $\mathbf{r}$ are the same. Hence, for the velocity-based realisation, (48), the linear controllers are obtained by simply "freezing" the scheduling variable as required.

The equivalent velocity-based realisation for the realisation, (42), depicted in figure 6 with $\beta_{i}(\mathbf{r})=\left[\begin{array}{llll}b_{i}^{1} & (\mathbf{r}) & \cdots & b_{i}^{m}(\mathbf{r})\end{array}\right]$, $\mathrm{i}=1, . . \mathrm{n}+1$, is

$$
\begin{aligned}
& {\left[\begin{array}{c}
\dot{\mathrm{w}}_{1} \\
\dot{\mathrm{w}}_{2} \\
\vdots \\
\dot{\mathrm{w}}_{\mathrm{n}-1} \\
\dot{\mathrm{w}}_{\mathrm{n}}
\end{array}\right]=\left[\begin{array}{cccccc}
-\mathrm{a}_{1} & 1 & 0 & \cdots & 0 & 0 \\
-\mathrm{a}_{2} & 0 & 1 & & 0 & 0 \\
\vdots & \vdots & \vdots & & \vdots & \vdots \\
-\mathrm{a}_{\mathrm{n}-1} & 0 & 0 & & 0 & 1 \\
-\mathrm{a}_{\mathrm{n}} & 0 & 0 & \cdots & 0 & 0
\end{array}\right]\left[\begin{array}{c}
\mathrm{w}_{1} \\
\mathrm{w}_{2} \\
\vdots \\
\mathrm{w}_{\mathrm{n}-1} \\
\mathrm{w}_{\mathrm{n}}
\end{array}\right]+\left[\begin{array}{ccc}
-\mathrm{a}_{1} \mathrm{~b}_{1}^{1}(\mathbf{r})+\mathrm{b}_{2}^{1}(\mathbf{r}) & \cdots & -\mathrm{a}_{1} \mathrm{~b}_{1}^{\mathrm{m}}(\mathbf{r})+\mathrm{b}_{2}^{\mathrm{m}}(\mathbf{r}) \\
-\mathrm{a}_{2} \mathrm{~b}_{1}^{1}(\mathbf{r})+\mathrm{b}_{3}^{1}(\mathbf{r}) & -\mathrm{a}_{2} \mathrm{~b}_{1}^{\mathrm{m}}(\mathbf{r})+\mathrm{b}_{3}^{\mathrm{m}}(\mathbf{r}) \\
\vdots & \vdots \\
-\mathrm{a}_{\mathrm{n}-1} \mathrm{~b}_{1}^{1}(\mathbf{r})+\mathrm{b}_{\mathrm{n}}^{1}(\mathbf{r}) & -\mathrm{a}_{\mathrm{n}-1} \mathrm{~b}_{1}^{\mathrm{m}}(\mathbf{r})+\mathrm{b}_{\mathrm{n}}^{\mathrm{m}}(\mathbf{r}) \\
-\mathrm{a}_{\mathrm{n}} \mathrm{b}_{1}^{1}(\mathbf{r})+\mathrm{b}_{\mathrm{n}+1}^{1}(\mathbf{r}) & \cdots & -\mathrm{a}_{\mathrm{n}} \mathrm{b}_{1}^{\mathrm{m}}(\mathbf{r})+\mathrm{b}_{\mathrm{n}+1}^{\mathrm{m}}(\mathbf{r})
\end{array}\right]\left[\begin{array}{c}
\dot{\mathrm{r}}_{1} \\
\vdots \\
\dot{\mathrm{r}}_{\mathrm{m}}
\end{array}\right]} \\
& \dot{\mathrm{y}}=\left[\begin{array}{lllll}
1 & 0 & \cdots & 0 & 0
\end{array}\right]\left[\begin{array}{c}
\mathrm{w}_{1} \\
\mathrm{w}_{2} \\
\vdots \\
\mathrm{w}_{\mathrm{n}-1} \\
\mathrm{w}_{\mathrm{n}}
\end{array}\right]+\left[\begin{array}{lll}
\mathrm{b}_{1}^{1}(\mathbf{r}) & \cdots & \mathrm{b}_{1}^{\mathrm{m}}(\mathbf{r})
\end{array}\right]\left[\begin{array}{c}
\dot{\mathrm{r}}_{1} \\
\vdots \\
\dot{\mathrm{r}}_{\mathrm{m}}
\end{array}\right]
\end{aligned}
$$

where

$$
\mathrm{b}_{\mathrm{j}}^{\mathrm{i}}(\mathbf{r})=\nabla_{\mathrm{r}_{\mathrm{i}}} \mathrm{B}_{\mathrm{j}}(\mathbf{r}), \mathrm{i}=1,2, . ., \mathrm{m}, \mathrm{j}=1,2, . ., \mathrm{n}+1
$$

When $a_{n}$ is non-zero, this reformulation is equivalent to an algebraic nonlinear state transformation. The strong correspondence to the "frozen" scheduling variable linear controllers is evident from comparing (51) to (39).

The equivalent velocity-based representation, for the realisation, (44), depicted in figure 7 with $\beta_{1}(\hat{\mathbf{r}})=\left[\begin{array}{llll}b_{1}^{1} & b_{1}^{2}(\hat{\mathbf{r}}) & \cdots & b_{1}^{\mathrm{m}}(\hat{\mathbf{r}})\end{array}\right]$ and $\beta_{\mathrm{i}}(\mathrm{y}, \hat{\mathbf{r}})=\left[\begin{array}{llll}b_{\mathrm{i}}^{1} & \mathrm{~b}_{\mathrm{i}}^{2}(\mathrm{y}, \hat{\mathbf{r}}) & \cdots & \mathrm{b}_{\mathrm{i}}^{\mathrm{m}}(\mathrm{y}, \hat{\mathbf{r}})\end{array}\right], \mathrm{i}=2, . . \mathrm{n}+1$, is

$$
\begin{aligned}
& {\left[\begin{array}{c}
\dot{\mathrm{w}}_{1} \\
\dot{\mathrm{w}}_{2} \\
\vdots \\
\dot{\mathrm{w}}_{\mathrm{n}-1} \\
\dot{\mathrm{w}}_{\mathrm{n}}
\end{array}\right]=\left[\begin{array}{cccccc}
-\mathrm{a}_{1}(\mathrm{y}, \hat{\mathbf{r}}) & 1 & 0 & \cdots & 0 & 0 \\
-\mathrm{a}_{2}(\mathrm{y}, \hat{\mathbf{r}}) & 0 & 1 & & 0 & 0 \\
\vdots & \vdots & \vdots & & \vdots & \vdots \\
-\mathrm{a}_{\mathrm{n}-1}(\mathrm{y}, \hat{\mathbf{r}}) & 0 & 0 & & 0 & 1 \\
-\mathrm{a}_{\mathrm{n}}(\mathrm{y}, \hat{\mathbf{r}}) & 0 & 0 & \cdots & 0 & 0
\end{array}\right]\left[\begin{array}{c}
\mathrm{w}_{1} \\
\mathrm{w}_{2} \\
\vdots \\
\mathrm{w}_{\mathrm{n}-1} \\
\mathrm{w}_{\mathrm{n}}
\end{array}\right]+\left[\begin{array}{ccc}
-\mathrm{a}_{1}\left(\mathrm{y}, \hat{\mathbf{r}} \mathrm{b}_{1}^{1}+\mathrm{b}_{2}^{1}\right. & \cdots & -\mathrm{a}_{1}(\mathrm{y}, \hat{\mathbf{r}}) \mathrm{b}_{1}^{\mathrm{m}}(\hat{\mathbf{r}})+\mathrm{b}_{2}^{\mathrm{m}}(\mathrm{y}, \hat{\mathbf{r}}) \\
-\mathrm{a}_{2}(\mathrm{y}, \hat{\mathbf{r}}) \mathrm{b}_{1}^{1}+\mathrm{b}_{3}^{1} & -\mathrm{a}_{2}(\mathrm{y}, \hat{\mathbf{r}}) \mathrm{b}_{1}^{\mathrm{m}}(\hat{\mathbf{r}})+\mathrm{b}_{3}^{\mathrm{m}}(\mathrm{y}, \hat{\mathbf{r}}) \\
\vdots & \vdots \\
-\mathrm{a}_{\mathrm{n}-1}(\mathrm{y}, \hat{\mathbf{r}}) \mathrm{b}_{1}^{1}+\mathrm{b}_{\mathrm{n}}^{1} & -\mathrm{a}_{\mathrm{n}-1}(\mathrm{y}, \hat{\mathbf{r}}) \mathrm{b}_{1}^{\mathrm{m}}(\hat{\mathbf{r}})+\mathrm{b}_{\mathrm{n}}^{\mathrm{m}}(\mathrm{y}, \hat{\mathbf{r}}) \\
-\mathrm{a}_{\mathrm{n}}(\mathrm{y}, \hat{\mathbf{r}}) \mathrm{b}_{1}^{1}+\mathrm{b}_{\mathrm{n}+1}^{1} & \cdots & -\mathrm{a}_{\mathrm{n}}(\mathrm{y}, \hat{\mathbf{r}}) \mathrm{b}_{1}^{\mathrm{m}}(\hat{\mathbf{r}})+\mathrm{b}_{\mathrm{n}+1}^{\mathrm{m}}(\mathrm{y}, \hat{\mathbf{r}})
\end{array}\right]\left[\begin{array}{c}
\dot{\mathrm{r}}_{1} \\
\vdots \\
\dot{\mathrm{r}}_{\mathrm{m}}
\end{array}\right]} \\
& \dot{\mathrm{y}}=\mathrm{d}(\mathrm{y})\left[\begin{array}{lllll}
1 & 0 & \cdots & 0 & 0
\end{array}\right]\left[\begin{array}{c}
\mathrm{w}_{1} \\
\mathrm{w}_{2} \\
\vdots \\
\mathrm{w}_{\mathrm{n}-1} \\
\mathrm{w}_{\mathrm{n}}
\end{array}\right]+\mathrm{d}(\mathrm{y})\left[\begin{array}{lll}
\mathrm{b}_{1}^{1} & \cdots & \mathrm{b}_{1}^{\mathrm{m}}(\hat{\mathbf{r}})
\end{array}\right]\left[\begin{array}{c}
\dot{\mathrm{r}}_{1} \\
\vdots \\
\dot{\mathrm{r}}_{\mathrm{m}}
\end{array}\right]
\end{aligned}
$$

where

$$
\begin{aligned}
& a_{j}(y, \hat{\mathbf{r}})=-\nabla_{z} B_{j+1}\left(G^{-1}(y), \hat{\mathbf{r}}\right), j=1,2, . ., n ; b_{1}^{i}(\hat{\mathbf{r}})=\nabla_{r_{i}} B_{1}(\hat{\mathbf{r}}), i=2,3, \ldots m ; \\
& b_{j}^{i}(y, \hat{\mathbf{r}})=\nabla_{r_{i}} B_{j}\left(G^{-1}(y), \hat{\mathbf{r}}\right), i=2,3, . . m, j=1,2, . . n ; d(y)=\nabla_{z} G\left(G^{-1}(y)\right)
\end{aligned}
$$

When $\mathrm{B}_{\mathrm{n}+1}(\bullet, \hat{\mathbf{r}})$ is invertible for all $\hat{\mathbf{r}}$, this representation is equivalent to an algebraic nonlinear state transformation of (44). The scheduling with respect to the output is no longer implicit but is now explicit by virtue of the invertibility of $\mathrm{G}(\bullet)$. The strong correspondence to the "frozen" scheduling variable linear controllers is again evident from comparing (52) to (39). 
Example Returning to the wind turbine Example in section 3.5, the first realisation, (46), can be recast as the velocitybased realisation,

$$
\left[\begin{array}{c}
\dot{\mathrm{w}}_{1} \\
\dot{\mathrm{w}}_{2} \\
\dot{\mathrm{w}}_{3} \\
\dot{\mathrm{w}}_{4}
\end{array}\right]=\left[\begin{array}{cccc}
-135.8 & 1 & 0 & 0 \\
-3818 & 0 & 1 & 0 \\
-26150 & 0 & 0 & 1 \\
-204000 & 0 & 0 & 0
\end{array}\right]\left[\begin{array}{c}
\mathrm{w}_{1} \\
\mathrm{w}_{2} \\
\mathrm{w}_{3} \\
\mathrm{w}_{4}
\end{array}\right]+\left[\begin{array}{c}
-135.8 \mathrm{~b}_{1}(\mathrm{r})+\mathrm{b}_{2}(\mathrm{r}) \\
-3818 \mathrm{~b}_{1}(\mathrm{r})+\mathrm{b}_{3}(\mathrm{r}) \\
-26150 \mathrm{~b}_{1}(\mathrm{r})+\mathrm{b}_{4}(\mathrm{r}) \\
-204000 \mathrm{~b}_{1}(\mathrm{r})+\mathrm{b}_{5}(\mathrm{r})
\end{array}\right] \dot{\mathrm{r}}, \quad \dot{\mathrm{y}}=\mathrm{w}_{1}+\mathrm{b}_{1}(\mathrm{r}) \dot{\mathrm{r}}
$$

where

$$
\begin{aligned}
& b_{1}(r)=4.82 \times 10^{-6} r^{2}-1.15 \times 10^{-3} r+1.042, b_{2}(r)=3.34 \times 10^{-5} r^{2}+1.68 \times 10^{-3} r+13.57, \\
& b_{3}(r)=2.78 \times 10^{-4} r^{2}+3.15 \times 10^{-2} r+146.33, b_{4}(r)=5.82 \times 10^{-4} r^{2}+0.37 r+676.16, \\
& b_{5}(r)=1.38 \times 10^{-3} r^{2}+1.93 r+2295.00
\end{aligned}
$$

Similarly, the second realisation, (48), can be recast as

$$
\left[\begin{array}{c}
\dot{\mathrm{w}}_{1} \\
\dot{\mathrm{w}}_{2}
\end{array}\right]=\left[\begin{array}{cc}
-\mathrm{a}_{1}(\mathrm{y}) & 1 \\
-\mathrm{a}_{2}(\mathrm{y}) & 0
\end{array}\right]\left[\begin{array}{c}
\mathrm{w}_{1} \\
\mathrm{w}_{2}
\end{array}\right]+\left[\begin{array}{c}
-0.00147 \mathrm{a}_{1}(\mathrm{y})+0.111 \\
-0.00147 \mathrm{a}_{2}(\mathrm{y})+1
\end{array}\right] \dot{\mathrm{r}}, \quad \dot{\mathrm{y}}=\mathrm{d}(\mathrm{y}) \mathrm{w}_{1}-\mathrm{d}(\mathrm{y}) 0.00147 \dot{\mathrm{r}}
$$

where

$$
\mathrm{a}_{1}(\mathrm{y})=-0.033 \mathrm{y}^{2}+0.750 \mathrm{y}+3.375, \mathrm{a}_{2}(\mathrm{y})=2.600 \mathrm{y}+58.040, \mathrm{~d}(\mathrm{y})=0.138 \mathrm{y}+0.298
$$

The correspondence to the original families of linear controllers, (41) and (40) respectively, is clear; in particular, compare (53b) to (41e) and (54b) to (40e).

Whilst, in general, the reformulation to velocity-based realisations increases the controller order, the realisations of figures 5 and 6 have the distinct advantage of being directly related to the members of the family of linear controllers. The associated family of linear controllers for the realisation, (51), now consists simply of (39) or, equivalently, the transfer functions, (38), with $d=1$ and $b_{j}^{i}=b_{j}^{i}\left(\mathbf{r}_{o}\right), i=1,2, . ., m ; j=1,2, . ., n+1$. The associated family for the realisation, (52), consists of (39) or, equivalently, (38), with $d=d\left(y_{o}\right), a_{j}=a_{j}\left(y_{o}, \hat{\mathbf{r}}_{o}\right), b_{1}^{i}=b_{1}^{i}\left(\hat{\mathbf{r}}_{o}\right), b_{j+1}^{i}=b_{j+1}^{i}\left(y_{o}, \hat{\mathbf{r}}_{o}\right)$, $\mathrm{i}=2, . . \mathrm{m}, \mathrm{j}=1, . . \mathrm{n}$.

\subsection{Integral Action}

The third issue, namely reconciling the equilibrium values of the controller input and output, is resolved by including integral action in the controller. As noted in section 2.2, $\mathbf{y}$ is an input to the plant and $\mathbf{r}$ is an output from the plant, the values of which, at an equilibrium operating point of the plant, are $\left(\mathbf{r}_{\mathbf{0}}(\pi), \mathbf{y}_{\mathbf{0}}(\boldsymbol{\pi})\right)$ with $\mathbf{r}_{\mathbf{0}}(\pi)$ dependent on $\mathbf{y}_{\mathbf{0}}(\pi)$ via the plant. However, since $\mathbf{r}$ is the controller input and $\mathbf{y}$ is the controller output, $\left(\mathbf{r}_{\mathbf{0}}(\pi), \mathbf{y}_{\mathbf{0}}(\pi)\right)$ must also be an equilibrium operating point of the controller with $\mathbf{y}_{\mathbf{0}}(\pi)$ dependent on $\mathbf{r}_{\mathbf{o}}(\boldsymbol{\pi})$ via the controller. Requiring consistency imposes, in general, a strong restriction on the allowable nonlinear controllers.

Fortunately, the control design task can usually be defined such that the controller acts upon an error which is, typically, required to be zero or small when in equilibrium. Since, in order to meet performance requirements, controllers often possess integral action, which ensures that the input must be zero in equilibrium, the former case is frequently encountered. In the latter case, the lack of precision in defining what is meant by small frequently enables the equilibrium value of the input, $\mathbf{r}$, to be redefined to be zero; that is, the change induced in the equilibrium operating point by this redefinition is typically sufficiently small that it is immaterial. When the input to the controller is zero in equilibrium, the $\mathbf{y}_{\mathbf{0}}(\boldsymbol{\pi})$ are no longer dependent on the $\mathbf{r}_{\mathbf{0}}(\boldsymbol{\pi})$, but can have any value. Hence, the consistency requirement on the equilibrium values of the input and output of the controller is not restrictive (Shamma 1988, in a rather more restricted context, make a similar observation regarding the role of integral action in gain-scheduled controllers).

\subsubsection{Velocity-based realisations revisited}

It is noted in section 4.2 that, for a velocity-based realisation to be viable, any unwanted constant displacement of the state or output, due to the differentiation of the input followed by the integration to determine the output from $\dot{\mathbf{y}}$, must be avoided. This can, also, be achieved by the inclusion of integral action in the controller.

Consider the simple nonlinear controller consisting of a single nonlinear gain together with integral action as depicted in figure 8a. The nonlinear component

$$
\mathrm{y}=\mathrm{G}(\mathrm{r})
$$


itself constitutes a nonlinear controller satisfying the extended local linear equivalence condition. The scheduling variable can be considered to be either the input, r, or, when the inverse of $\mathrm{G}(\bullet)$ exists, implicitly the output, y. Positioning the integrator before the nonlinear gain immediately makes both $\mathrm{r}$ and $\mathrm{r}$ available. The nonlinear controller, with $\mathrm{r}$ as the scheduling variable, can be recast as the velocity-based realisation, depicted in figure $8 \mathrm{~b}$,

$$
\dot{\mathrm{y}}=\nabla \mathrm{G}(\mathrm{r}) \dot{\mathrm{r}}
$$

The nonlinear controller, with y as the scheduling variable, can be recast as the velocity-based realisation, depicted in figure $8 \mathrm{c}$,

$$
\dot{y}=\mathrm{g}(\mathrm{y}) \dot{\mathrm{r}}, \quad \mathrm{g}(\mathrm{y})=\nabla \mathrm{G}\left(\mathrm{G}^{-1}(\mathrm{y})\right)
$$

In both figures $8 \mathrm{~b}$ and $8 \mathrm{c}$, the differentiation and integration, associated with the velocity-based realisation, do not appear explicitly but merely cause a repositioning of the pure integrator from before to after the nonlinear element. Note that in the realisation, (56), the scheduling variable, $r$, must be determined by integrating $\dot{\mathrm{r}}$, but in the realisation, (57), the scheduling variable, $y$, is directly available. Since the closed-loop system is stable, any error in the initial condition for y rapidly evolves out. The realisation, depicted in figure 8c, is a rather simple and effective means of realising a system consisting of a single nonlinear gain together with integral action. It is widely applicable; for example, in wind turbine control to cater for the variation in the aerodynamics with wind speed, see Leithead et al. (1992). (A somewhat more rigorous explanation for the remarkable effectiveness of this approach to scheduling the controller, when applied to wind turbines, is presented in Leith \& Leithead (1997b)).

The reason for adopting the velocity-based realisations is to avoid having to determine the nonlinear functions of the scheduling variable in the nonlinear controller from the relationships of their partial derivatives to the linear controllers' transfer function coefficients. Unfortunately, with the introduction of integral action, the velocity-based realisation no longer resolves this issue since the equilibrium operating points cannot be parameterised by the input, $\mathrm{r}_{\mathrm{o}}$. Consider the simple nonlinear controller, (55), without integral action and its velocity-based realisation with the scheduling variable $r$, (56). The equilibrium operating points, $\left(r_{0}, y_{0}\right)$, may be parameterised by $r_{o}$ or $y_{0}$. Hence, when designing the linear controllers (the gains) at the equilibrium operating points, it is possible to directly parameterise them by $r_{o}$ and so to directly determine $\nabla \mathrm{G}(\mathrm{r})$. However, when integral action is included and initially positioned, as above, before the nonlinear element, the equilibrium values of the input, $r_{0}$, are no longer related to the plant input but are related to the controller output, $\mathrm{y}_{\mathrm{o}}$, via the nonlinear controller itself. Hence, when designing the linear controllers, it is not possible to directly parameterise the gains by $\mathrm{r}_{\mathrm{o}}$. Instead, the gains must first be parameterised by $\mathrm{y}_{\mathrm{o}}$, say $\mathrm{g}_{\mathrm{yo}}$. From (55) and (57), it then follows that

$$
\nabla \mathrm{G}(\mathrm{r})=\mathrm{g}(\mathrm{G}(\mathrm{r}))
$$

with

$$
\mathrm{G}^{-1}(\rho)=\int_{\mathrm{o}}^{\rho} \frac{1}{\mathrm{~g}(\mathrm{~s})} \mathrm{ds}, \quad \mathrm{g}(\mathrm{s})=\mathrm{g}_{\mathrm{s}}
$$

and integration of the coefficients, in this case the inverse of the gain, cannot be avoided.

Of course, the two realisations, (42)/(51) and (44)/(52), can be treated in a similar manner to the above simple nonlinear controller consisting of a single nonlinear gain together with integral action. Adoption of the velocity-based realisations, (51) and (52), merely causes the repositioning of the integral action from before to after the nonlinear controller. Except for realisation (52), when specialised to the SISO case, the scheduling variable still requires to be determined by integrating $\dot{\mathbf{r}}$ and the inverses of the controller gains still require to be integrated to obtain a direct parameterisation of the linear controllers in terms of the input, $\mathbf{r}$.

Example Returning to the wind turbine Example in section 3.5, the fixed component, $\mathrm{K}(\mathrm{s})$ or $\mathrm{K}^{\prime}$ (s), which is positioned before the gain-scheduled component, $\mathrm{C}_{\pi}$ or $\mathrm{C}_{\pi}{ }^{\prime}$, includes integral action. Hence, the first velocity-based realisation, (53), can be reformulated as depicted in figure $9 \mathrm{a}$ with the coefficients $\mathrm{b}_{\mathrm{i}}(\mathrm{r}) \mathrm{i}=1, . .5$, defined by (53b). The parameterisation of the $b_{i}(r),(53 b)$, and equivalently the $b_{i}(\pi),(41 e)$, are obtained by a similar procedure to that described above for the simple nonlinear controller consisting of a nonlinear gain together with integral action. The relationship between the $r_{o}$ and $y_{o}$ is $r_{o}=-1.42 y_{o}{ }^{2}+88.51 y_{o}-311.72$. The scheduling variable, $r$, is obtained by integrating $\dot{r}$. A stable weak feedback from the controller output is employed to ensure that any error in the initial condition for $r$ evolves out.

The second velocity-based realisation, (54), can be reformulated as depicted in figure $9 \mathrm{~b}$, with the coefficients $\mathrm{a}_{1}(\mathrm{y})$, $\mathrm{a}_{2}(\mathrm{y})$ and $\mathrm{d}(\mathrm{y})$ defined by (54b). This realisation is the one reported in Leith \& Leithead (1996). Both of these realisations, figure $9 \mathrm{a}$ and figure $9 \mathrm{~b}$, have been implemented on a detailed non-linear simulation of the wind turbine and their performance assessed: they are found to be equally effective.

It is well known empirically, although rarely reported in the literature (Lawrence \& Rugh 1995), that it is beneficial, where possible, to position a pure integrator at the output of a gain-scheduled controller. The foregoing discussion provides analytic support for this heuristic rule. 


\subsection{Direct realisations}

In sections 4.1-4.3, several issues related to the practical design of nonlinear controllers satisfying the extended local linear equivalence conditions are investigated and various ways of resolving them determined. Of course, in any particular application, the most appropriate methods for the context should be adopted. However, a realisation which automatically resolves all of these issues would be useful. Just such a realisation for MIMO controllers is developed below.

The general structure of the realisation is depicted in figure 10a. The components $\mathrm{C}_{\mathrm{po}}$ and $\mathrm{C}_{\mathrm{pr}}$ are fixed, with all of the scheduled components in $\mathrm{C}_{\rho}$, and the controller is assumed to include integral action positioned as shown explicitly in figure 10a. Without loss of generality, $\mathrm{C}_{\mathrm{po}}$ and $\mathrm{C}_{\rho}$ are assumed square; that is, any non-squareness is incorporated into $\mathrm{C}_{\mathrm{pr}}$. Because the input to the controller is zero at the equilibrium operating points, the maximum number of scheduling variables which can be accommodated by the realisation of figure 10a is $\mathrm{p}$, the degree of the output; that is, the degree of $\mathbf{y}$ since $C_{p o}$ is square. The component $C_{p o}$ is chosen such that the parameterisation of the equilibrium operating points by the elements of $\mathbf{y}$ is compatible, in as natural a manner as possible, with the scheduling requirements. Any necessary non-singular transformation of the outputs is incorporated into $\mathrm{C}_{\mathrm{po}}$.

A further requirement on the assignment of components of the controller to $\mathrm{C}_{\mathrm{pr}}$ and $\mathrm{C}_{\mathrm{po}}$ is to ensure that, as discussed in section 4.1, the spectra of the scheduling variable has suitable bandwidth. For the wind turbine Example in section 3.5, it is appropriate to schedule the controller with respect to the pitch angle of the rotor blades. When the bandwidth of the actuator is large, a suitable alternative to the pitch angle is the pitch demand to the actuator; that is, the controller output. However, for some wind turbines, particularly large-scale machines, the bandwidth of the actuator can be very low (see, for example, Leith \& Leithead 1997b and the references therein). Consequently, the rate of variation of the pitch demand can be significantly faster than the pitch angle itself. The remedy, adopted in Leith \& Leithead (1997b) (albeit, more rigorously justified), is to choose $C_{p o}$ to be an inverse or pseudo-inverse, $A^{-1}$, of the actuator dynamics. When this remedy is applied to the simple controller of figure $8 \mathrm{c}$, it is amended as shown in figure $8 \mathrm{~d}$. The integral action is exploited by permitting $\mathrm{A}^{-1}$ to be a simple lead term, with transfer function ( $\left.\mathrm{s}+\mathrm{b}\right) / \mathrm{b}$, since their combined transfer function is proper.

The nonlinear component, $\mathrm{C}_{\rho}$, of the controller is scheduled with respect to the input, $\mathrm{r}$. With this choice of scheduling variable, the realisation, satisfying the extended local linear equivalence condition, for $\mathrm{C}_{\rho}$ and the corresponding family of linear controllers have the most convenient form, see section 4.1, and the restrictions on the class of valid inputs and initial conditions is reduced to an absolute minimum, see section 3.3. Interpreting $\mathrm{C}_{\rho}$ to consist of p multi-input single-output nonlinear controllers, the realisation for each conforms to that of figure 4 .

For each of the $\mathrm{p}$ multi-input single-output nonlinear controllers, let the variable, $\mathrm{z}$, be the scalar function, $\mathrm{B}_{\mathrm{n}+1}(\mathbf{r})$, of the input, $\mathbf{r}$. To distinguish this relationship for the different multi-input single-output controllers, an index $\mathrm{k}$ is used; for the controller relating $\mathbf{r}$ to the $\mathrm{k}^{\text {th }}$ element of $\mathbf{y}, \mathrm{z}^{\mathrm{k}}=\mathrm{B}^{\mathrm{k}}{ }_{\mathrm{n}+1}(\mathbf{r})$. Let $\mathbf{z}$ be the vector whose elements are the $\mathrm{z}^{\mathrm{k}}$, and $\mathbf{B}(\mathbf{r})$ be the vector whose elements are the $\mathrm{B}_{\mathrm{n}+1}^{\mathrm{k}}(\mathbf{r})$, then

$$
\mathbf{z}=\mathbf{B}(\mathbf{r})
$$

Assume that the inverse, $\mathbf{B}^{-1}(\bullet)$, of $\mathbf{B}(\bullet)$ exists. The controller of figure 10a can then be reformulated as shown in figure 10b. The nonlinear controller, $\mathrm{C}_{\rho}^{*}$, consists of $\mathrm{p}$ multi-input single-output nonlinear controllers with the realisation depicted in figure 11, where, for the $\mathrm{k}^{\text {th }}$ multi-input single-output nonlinear controller, $\mathbf{c}$ is the vector for which the $\mathrm{k}^{\text {th }}$ element is unity, all other elements being zero, and $\hat{B}_{i}(\bullet)=B_{i}^{k}\left(\mathbf{B}^{-1}(\bullet)\right), \mathrm{i}=1, . . \mathrm{n}$. It should noted that, due to the definition of $\mathbf{B}(\bullet)$, the elements of $\mathbf{z}$ are equal to the scaled elements of $\mathbf{y}$ (by the $\mathrm{a}_{\mathrm{n}}$ ) at an equilibrium operating point.

The equivalent velocity-based realisation for the multi-input single-output nonlinear controller of figure 11 is (51) with the coefficients defined by

$$
\mathrm{b}_{\mathrm{j}}^{\mathrm{i}}(\mathbf{z})=\nabla_{\mathrm{z}_{\mathrm{i}}} \hat{\mathrm{B}}_{\mathrm{j}}(\mathbf{z}), \mathrm{i}=1, . . \mathrm{m}, \mathrm{j}=1, . . \mathrm{n}, \quad \mathrm{b}_{\mathrm{n}+1}^{\mathrm{i}}=\mathrm{c}_{\mathrm{i}}, \mathrm{i}=1, . ., \mathrm{m}
$$

where $c_{i}$ is the $i^{\text {th }}$ element of c. This realisation is depicted in figure 12 , which corresponds to figure 6 , with $\hat{\beta}_{\mathbf{i}}(\mathbf{z})=\left[\begin{array}{llll}b_{i}^{1}(\mathbf{z}) & \ldots & b_{i}^{m}(\mathbf{z})\end{array}\right], i=1, . . n$. In figure 12 , the feedback loops have all been shifted one integration to the right. It should be noted that $\mathbf{z}$ is available internally to the realisation depicted in figure 12 and, therefore, only $\mathbf{z}$ is needed as an input to $\mathrm{C}_{\rho}{ }^{*}$. Since the closed-loop system would be stable, any error in the initial conditions for the integration of $\dot{\mathbf{z}}$ rapidly evolves out.

The nonlinear controller, consisting of the function, $\mathbf{B}(\bullet)$, together with the integral action, can also be reformulated in a similar manner to the simple scalar case considered in section 4.3 as the velocity-based realisation depicted in figure 13, where $\hat{\mathbf{B}}(\mathbf{z})=\left[\hat{\mathrm{B}}_{\mathrm{ij}}(\mathbf{z})\right]=\left[\nabla_{\mathrm{r}_{\mathrm{j}}} \mathrm{B}_{\mathrm{i}}\left(\mathbf{B}^{-1}(\mathbf{z})\right)\right]$. Since $\mathbf{z}$ is not required as an input to $\mathrm{C}_{\rho}{ }^{*}$, the integral action in the velocity-based realisation can be omitted when the complete controller has the realisation depicted in figure $11 \mathrm{c}, \mathrm{C}_{\rho}{ }^{*}$ being realised as in figure 12. Moreover, since $\mathbf{z}$ has been chosen so that, at an equilibrium operating point, the 
elements of $\mathbf{z}$ and $\mathbf{y}$ are related by simple scaling factors, the equilibrium operating points, and so the family of linear controllers, may be parameterised equally well by $\mathbf{z}$ or $\mathbf{y}$.

A practical procedure, based on the foregoing (with minor modifications so that the parameterisations of the equilibrium operating points with respect to $\mathbf{y}$ and $\mathbf{z}$ are identical), for the design of MIMO gain-scheduled controllers is as follows. Design a family of linear controllers, parameterised by the output, $\mathbf{y}$, such that the denominator coefficients of all the elements in a row of the transfer function matrix, $\mathbf{G}(\mathrm{s})$, are the same and fixed, but the numerator coefficients may vary essentially independently. For each member of the family, determine the two matrices, G(0) and $\mathbf{H}(\mathrm{s})=\mathbf{G}(\mathrm{s}) \mathbf{G}^{-1}(0)$. $\mathbf{H}(\mathrm{s})$ is implemented directly as $\mathrm{p}$ multi-input single-output nonlinear controllers realised as in figure 12 , with the unity element of $\mathbf{c}$ changed to $a_{n}$ and the $b_{j}^{i}(\mathbf{z})$ the numerator coefficients of $\mathbf{H}(\mathrm{s})$ parameterised with respect to $\mathbf{z} . \mathbf{G}(0)$ is implemented directly as the gain matrix, $\hat{\mathbf{B}}(\mathbf{z})$, of figure 10 c, with the elements of $\hat{\mathbf{B}}(\mathbf{z})$ identical to the elements of $\mathbf{G}(0)$ parameterised with respect to $\mathbf{z}$.

Example Returning to the wind turbine Example in section 3.5, the family of linear controllers $(41 \mathrm{~b}), \mathrm{C}_{\pi}{ }^{\prime}(\mathrm{s}), \mathrm{re}-$ parameterised in terms of $\pi$, the equilibrium value of the pitch angle of the turbine rotor blades (equivalently, the controller output), is

$$
C^{\prime}(s)=\frac{b_{1}(\pi) s^{4}+b_{2}(\pi) s^{3}+b_{3}(\pi) s^{2}+b_{4}(\pi) s+b_{5}(\pi)}{s^{4}+135.8 s^{3}+3818 s^{2}+26150 s+204000}
$$

where

$$
\begin{aligned}
& \mathrm{b}_{1}(\pi)=0.0119 \pi^{2}-0.0863 \pi+1.128, \mathrm{~b}_{2}(\pi)=0.0666 \pi^{2}+0.307 \pi+10.923, \\
& \mathrm{~b}_{3}(\pi)=0.532 \pi^{2}+4.010 \pi+119.269, \mathrm{~b}_{4}(\pi)=62.543 \pi+418.033, \mathrm{~b}_{5}(\pi)=180.49 \pi+1591.50
\end{aligned}
$$

Hence,

$$
\begin{aligned}
& \mathrm{C}^{\prime}(0)=\mathrm{b}_{5}(\pi) / 204000 \\
& \mathrm{C}^{\prime}(\mathrm{s}) / \mathrm{C}^{\prime}{ }_{\pi}(0)=\frac{\hat{\mathrm{b}}_{1}(\pi) \mathrm{s}^{4}+\hat{\mathrm{b}}_{2}(\pi) \mathrm{s}^{3}+\hat{\mathrm{b}}_{3}(\pi) \mathrm{s}^{2}+\hat{\mathrm{b}}_{4}(\pi) \mathrm{s}+204000}{\mathrm{~s}^{4}+135.8 \mathrm{~s}^{3}+3818 \mathrm{~s}^{2}+26150 \mathrm{~s}+204000}
\end{aligned}
$$

with

$$
\hat{b}_{i}(\pi)=204000 b_{i}(\pi) / b_{5}(\pi), \quad i=1,2,3,4
$$

Following the above procedure, the realisation of the gain-scheduled nonlinear controller is that shown in figure 14. Note, that the varying coefficients employed in the nonlinear realisation are related directly to the corresponding coefficients of the linear family, (64); in fact, they are identical, attaining the value of the coefficients at the equilibrium operating point currently indicated by the value of the scheduling variable.

\section{Conclusions}

In this paper, the extended local linear equivalence condition of Leith \& Leithead (1996) is extended to encompass MIMO gain-scheduled nonlinear controllers. The extended local linear equivalence condition minimises the controller nonlinearity and, thereby, maximises the class of inputs and initial conditions for which the dynamic properties of the gain-scheduled nonlinear controller are similar to those of the members of the family of linear controllers on which it is based. Indeed, when the controller satisfies the extended local linear equivalence condition and the scheduling variable is selected to be the input to the gain-scheduled controller, there is no restriction on the initial conditions and the situation is exactly analogous to the linear time-varying case. The class of allowable inputs and initial conditions is, in particular, considerably greater than when the local linear equivalence condition of Lawrence \& Rugh (1995) at equilibrium operating points alone is satisfied.

Although the dynamic behaviour of the nonlinear controller must, as usual, be confirmed by analysis and/or simulation, the extended local linear equivalence condition does facilitate the design of gain-scheduled controllers by providing strong guidance to the most effective way of realising the controller. Several different classes of realisations, satisfying the extended local linear equivalence condition, are investigated and it is determined that the issues central to the practical design of gain-scheduled controllers can be resolved by these realisations. The design of the gainscheduled nonlinear controllers is facilitated by several realisations for which linear controllers are obtained by "freezing" the scheduling variable at its current value; that is, the varying coefficients of the nonlinear controller correspond precisely to the coefficients of the linear family, parameterised by the scheduling variable, on which the gain-scheduled design is based. Integral action has an important role in these direct realisations. In addition, it permits the equilibrium relationships, imposed by the plant, between the controller input and output to be accommodated in an elegant manner. The analysis, therefore, provides analytic support for the benefits, previously observed in practice, of including integral action in a gain-scheduled controller.

Whilst extended local linear equivalence might appear to be a rather strong condition, it is shown that it is possible to realise, with few exceptions, all gain-scheduled controllers as nonlinear controllers satisfying the extended local linear equivalence condition and requiring the controller to be so realised is not at all restrictive. 


\section{Acknowledgement}

D.J.Leith gratefully acknowledges the generous support provided by the Royal Society for the work presented.

\section{References}

ASTROM, K.J., WITTENMARK, B., 1989, Adaptive Control. (Addison-Wesley).

DESOER, C.A., 1969, Slowly Varying Controller dx/dt=A(t)x. IEEE Transactions on Automatic Control, 14, 780-781.

KAMINER, I., PASCOAL, A., KHARGONEKAR, P., COLEMAN, E., 1995, A Velocity Algorithm for the Implementation of Gain-Scheduled Controllers. Automatica, 31, 1185-1191.

LAWRENCE, D.A., RUGH, W.J., 1995, Gain Scheduling Dynamic Linear Controllers for a Nonlinear Plant. Automatica, 31, 381-390.

LEITH, D.J., LEITHEAD, W.E., 1994, Application of Nonlinear Control to a HAWT. Proceedings of the 3rd IEEE Conference on Control Applications, Glasgow.

LEITH, D.J., LEITHEAD, W.E., 1996, Appropriate Realisation of Gain Scheduled Controllers with Application to Wind Turbine Regulation. International Journal of Control, 65, 223-248.

LEITH, D.J., LEITHEAD, W.E., 1997a, Comments on 'Gain Scheduling Dynamic Linear Controllers for a Nonlinear Plant'. Automatica, to appear.

LEITH, D.J., LEITHEAD, W.E., 1997b, Implementation of Wind Turbine Controllers. International Journal of Control, 66, 349-380.

LEITHEAD,W.E., DE LA SALLE, S.A., REARDON, D., 1992, Classical Control of Active Pitch Regulation of Constant Speed HAWTs. International Journal of Control, 55, 845-876.

RUGH, W.J., 1991, Analytical Framework for Gain-Scheduling. IEEE Control Systems Magazine, 11, 79-84.

SHAMMA, J.S., 1988, Analysis and Design of Gain-Scheduled Control Systems (Ph.D. Thesis, Massachusetts Institute of Technology).

SHAMMA, J.S., ATHANS, M., 1990, Analysis of Gain Scheduled Control for Nonlinear Plants. IEEE Transactions on Automatic Control, 35, 898-907.

SHAMMA, J.S., ATHANS, M., 1992, Gain Scheduling: Potential Hazards and Possible Remedies. IEEE Control Systems Magazine, 12, 101-107. 
Figure 1 Nominal neighbourhoods for system satisfying the local linear equivalence condition.

Figure 2 Nominal neighbourhoods for system satisfying the extended local linear equivalence condition.

Figure 3 Different realisations of Example controller.

Figure 4 Realisation of m-input single-output controller, satisfying extended local linear equivalence condition, scheduled on input, $\mathbf{r}$.

Figure 5 Realisation of m-input single-output controller, satisfying extended local linear equivalence condition, scheduled on m-1 elements, $\hat{\mathbf{r}}$, of the inputs and implicitly on the output, $\mathrm{y}$.

Figure 6 Velocity-based realisation of m-input single-output controller, satisfying extended local linear equivalence condition, scheduled on input,. $\mathbf{r}$.

Figure 7 Velocity-based realisation of m-input single-output controller, satisfying extended local linear equivalence condition, scheduled on m-1 elements, $\hat{\mathbf{r}}$, of the inputs and explicitly on the output, $\mathrm{y}$.

Figure 8 Nonlinear controller realisations for family of linear controllers consisting simply of a varying gain together with integral action.

Figure 9 Velocity-based realisation for wind turbine controller example.

Figure 10 MIMO nonlinear controller realisations

Figure 11 Multi-input single-output controller realisation corresponding to $C_{\rho}^{*}$ in figure $11 \mathrm{~b}$

Figure 12 Velocity-based realisation of multi-input single-output controller of figure 12.

Figure 13 Velocity-based realisation corresponding to $\mathbf{B}(\mathbf{r})$ in figure $11 \mathrm{~b}$.

Figure 14 Direct realisation of wind turbine controller example. 
Figure 1

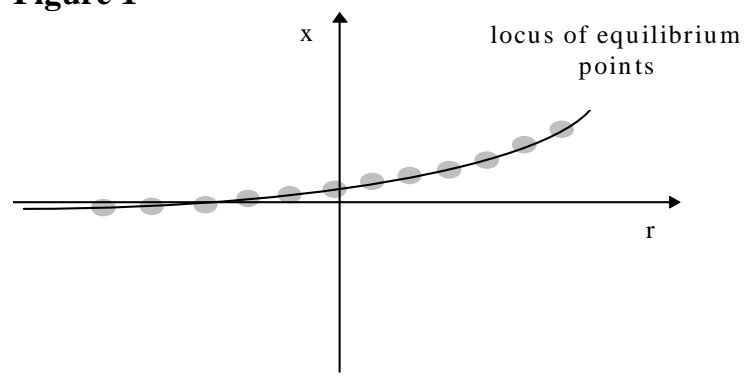

Figure 2

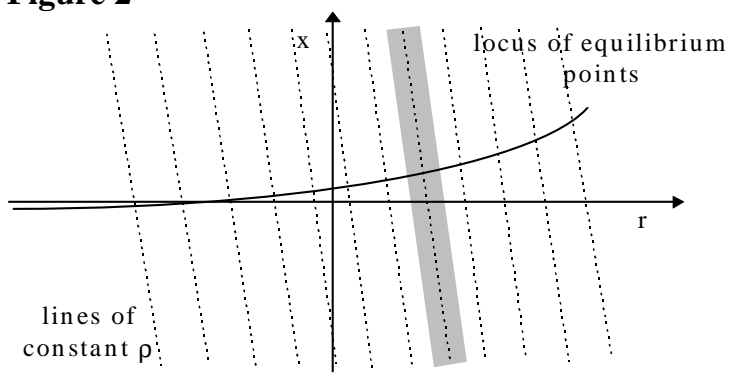

Figure 3a

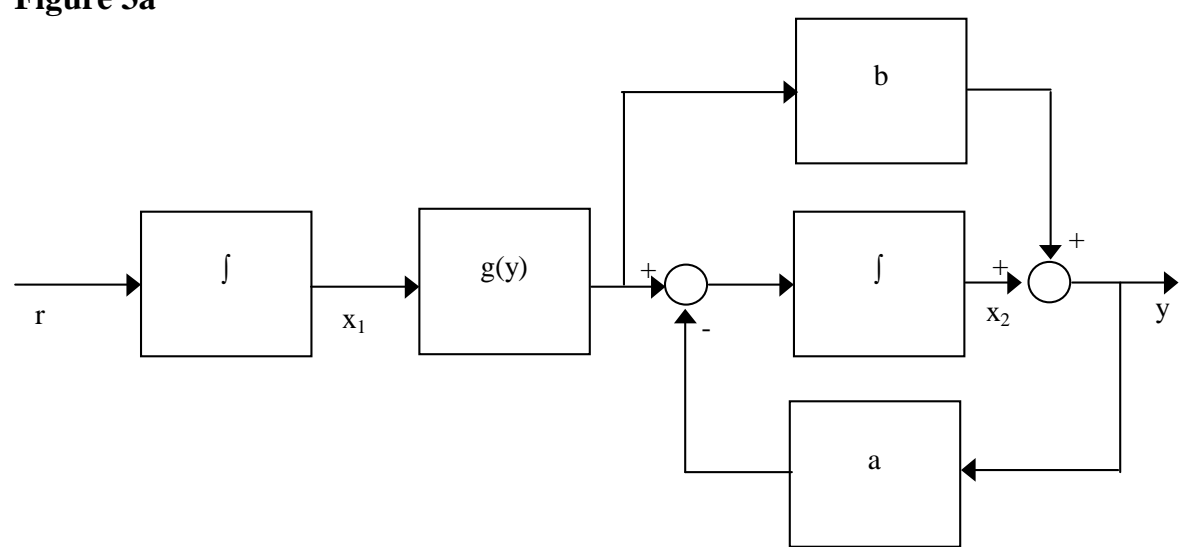

Figure 3b

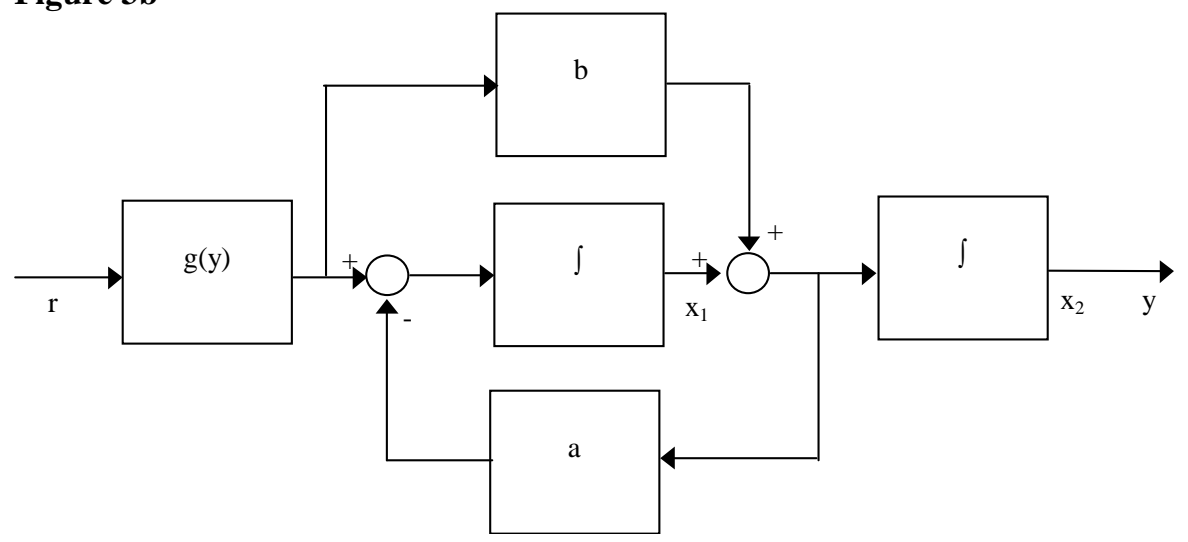

Figure 3c

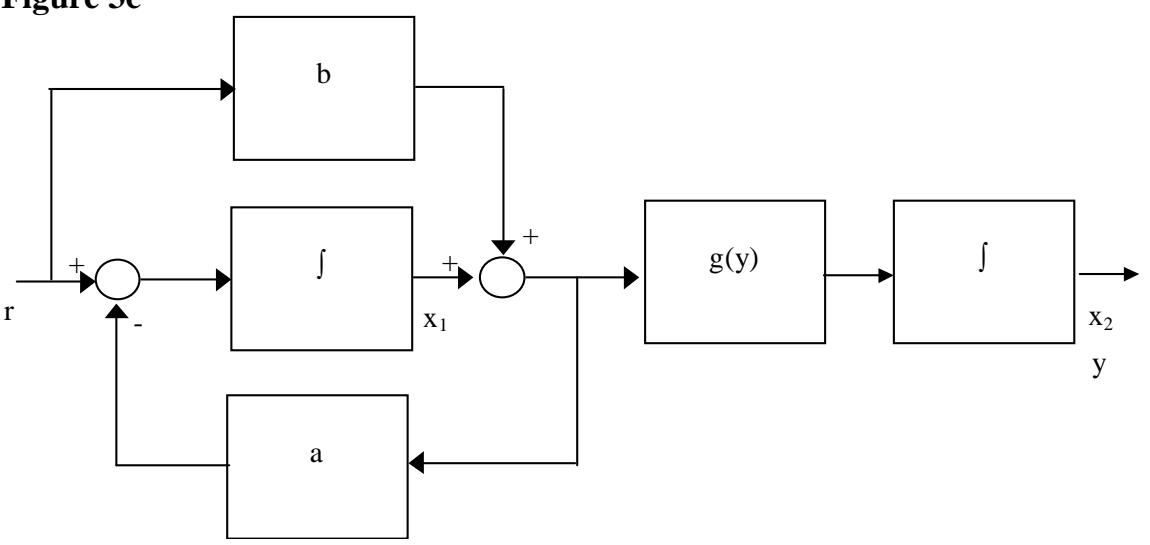




\section{Figure 4}

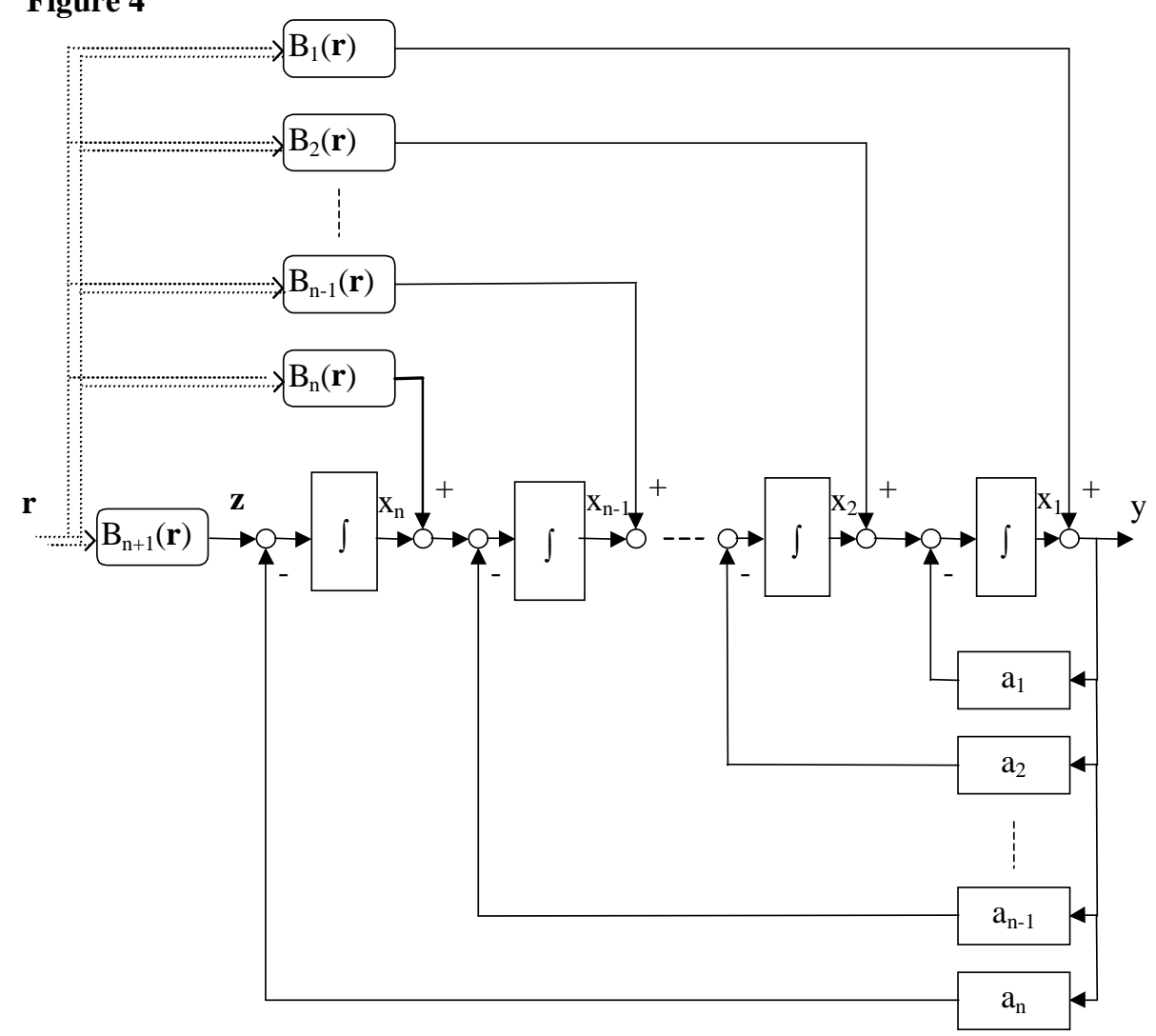

Figure 5

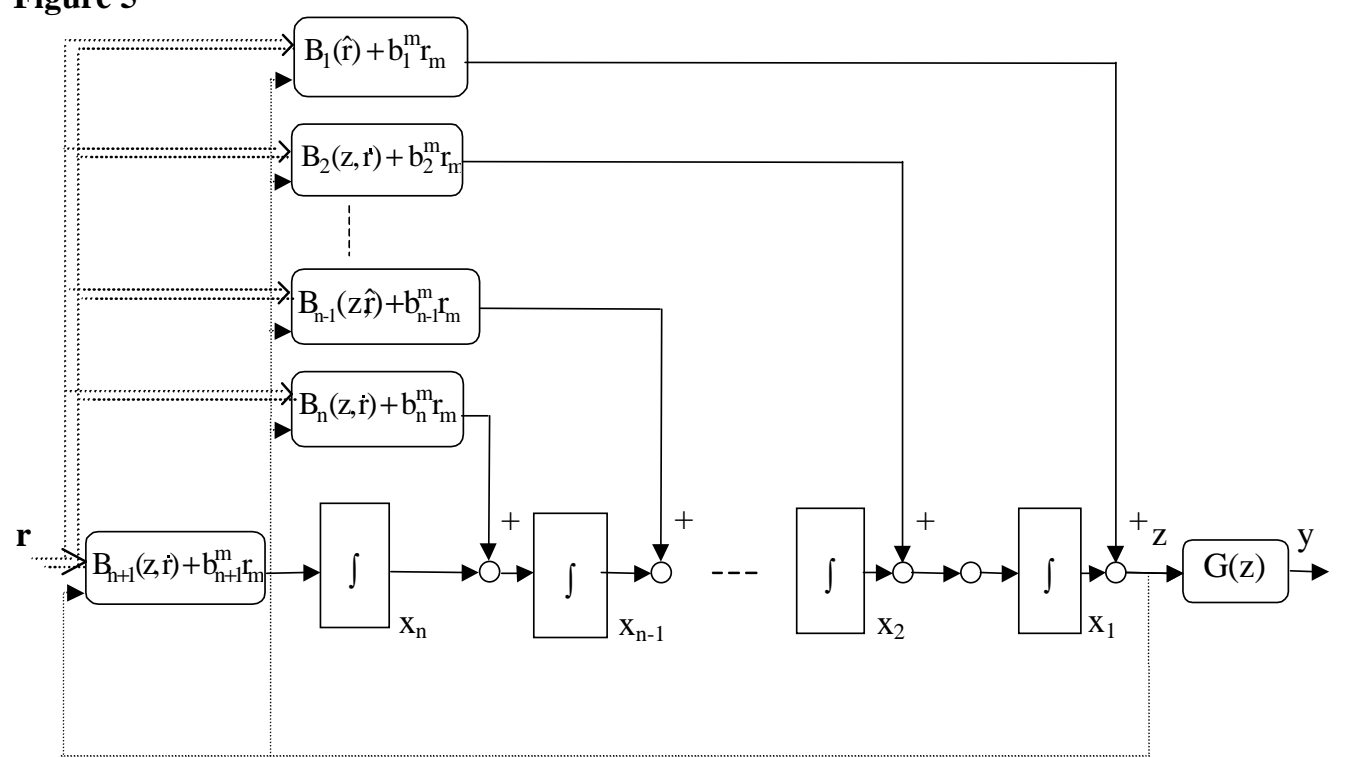




\section{Figure 6}

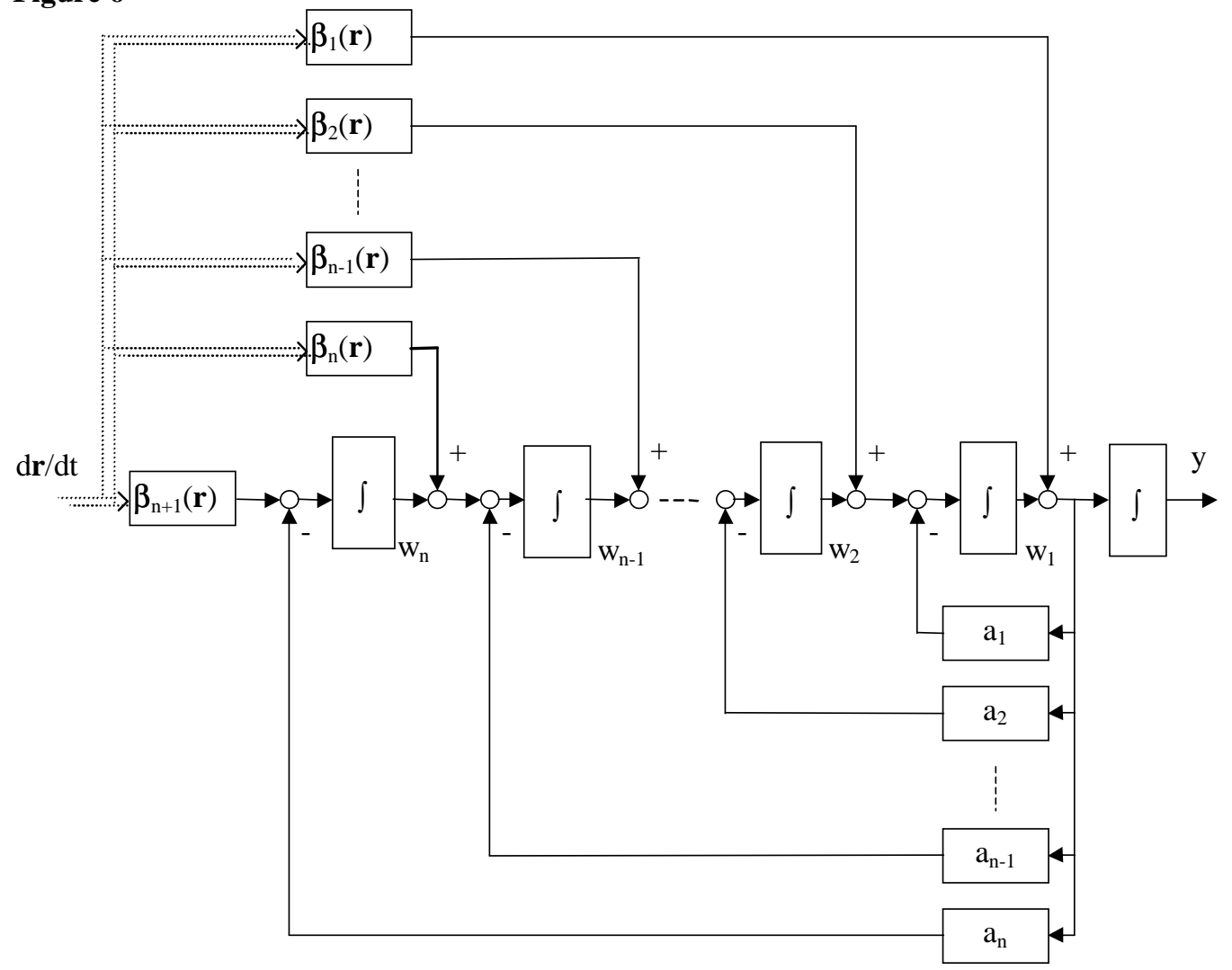

Figure 7

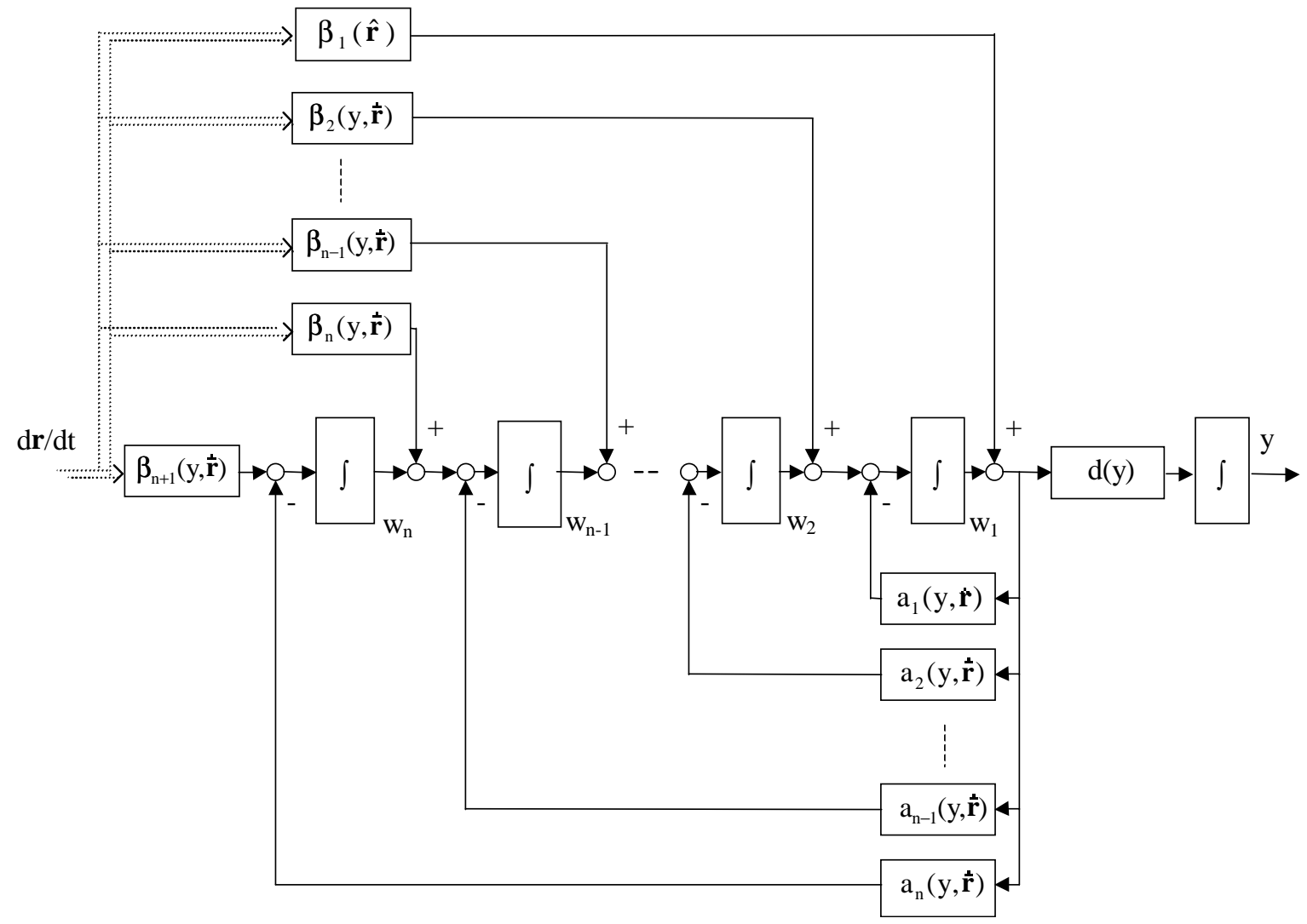


Figure 8a

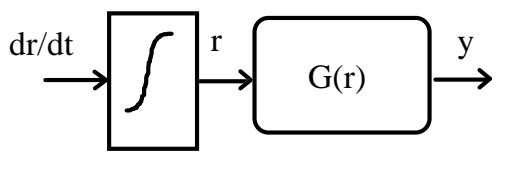

Figure 8b

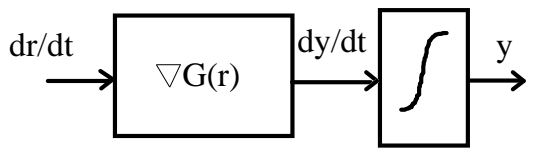

Figure 8c

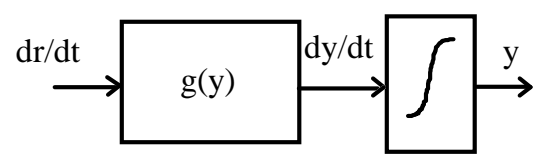

Figure 8d

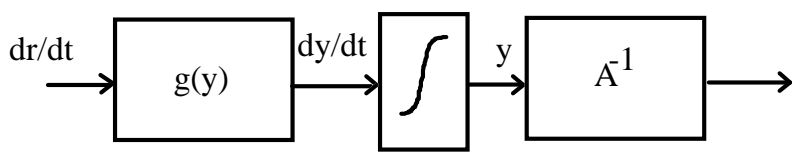

Figure 9a

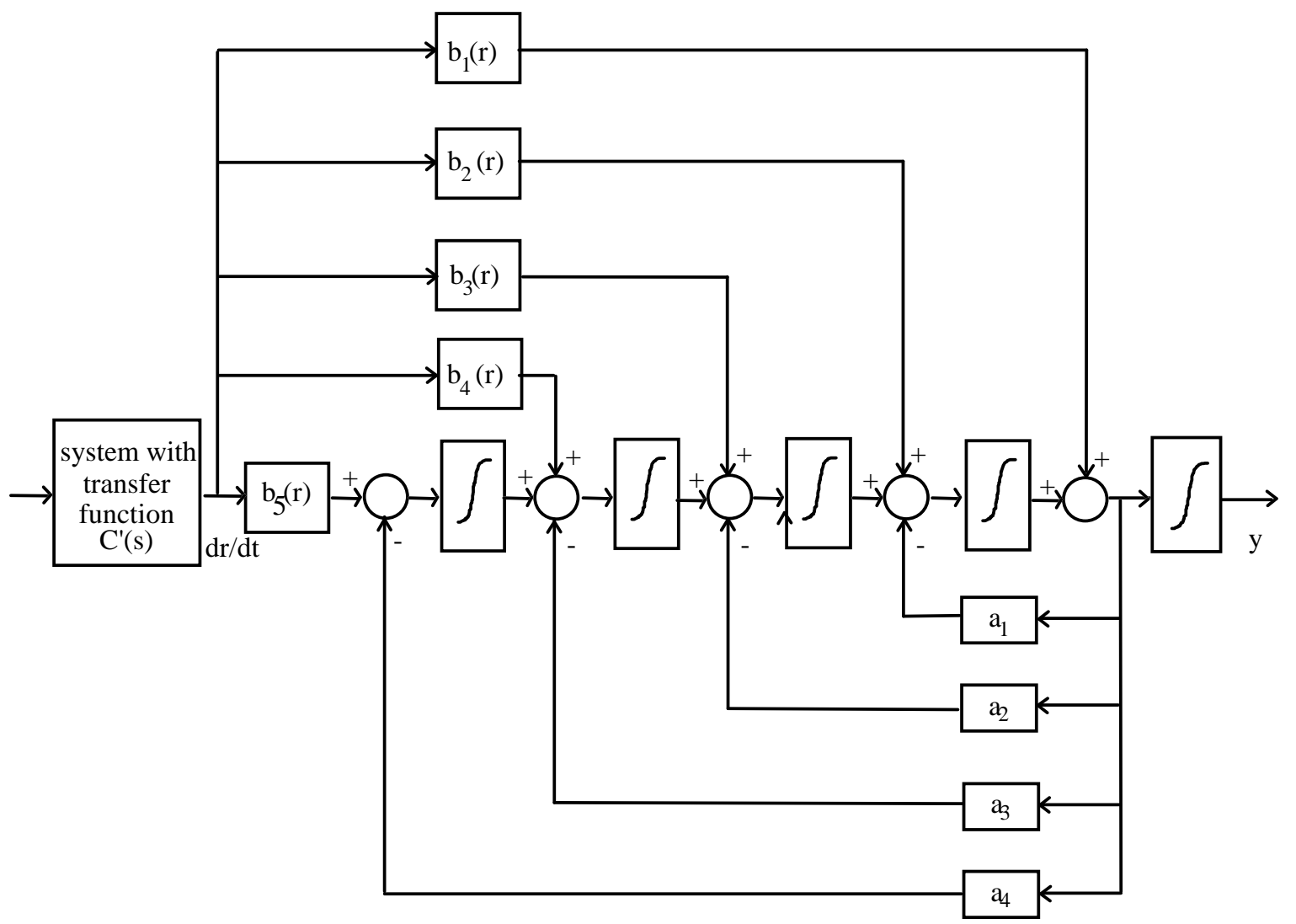

Figure 9b

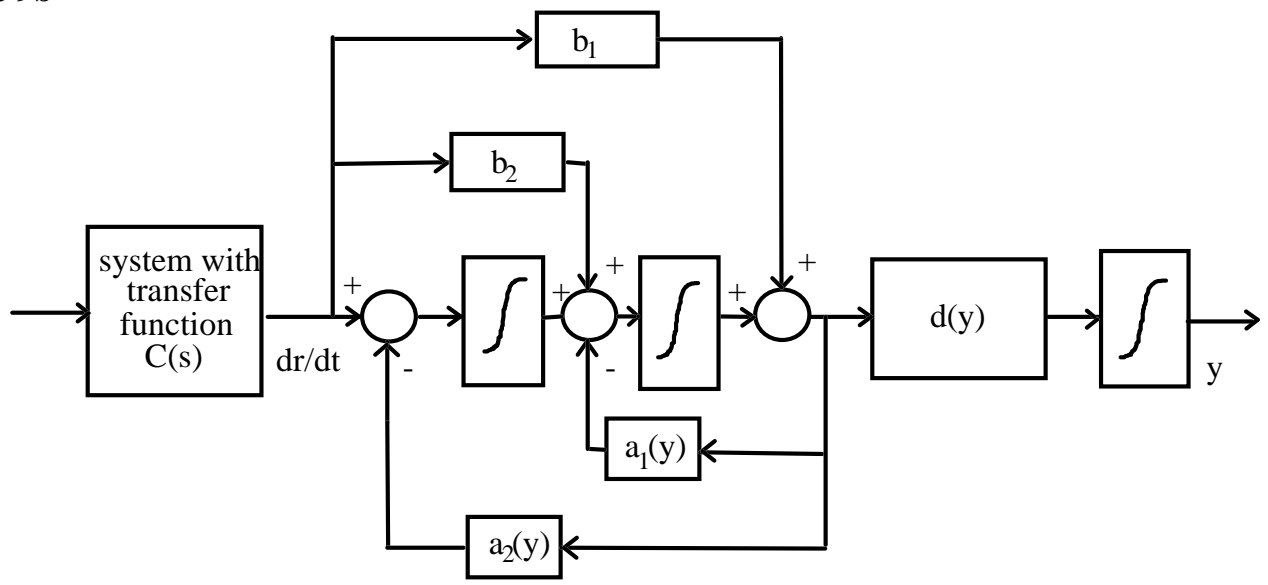


Figure 10a

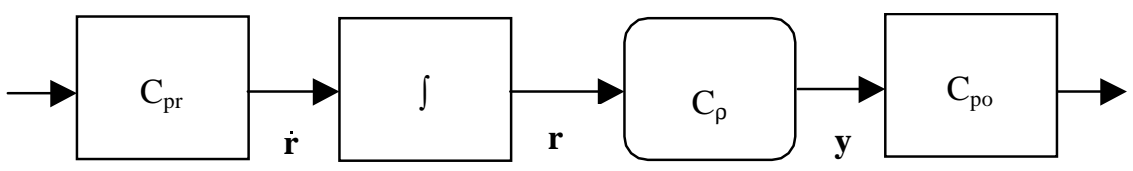

Figure 10b

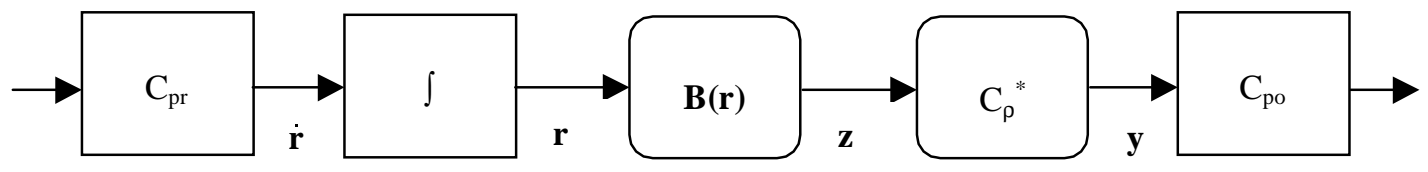

Figure 10c

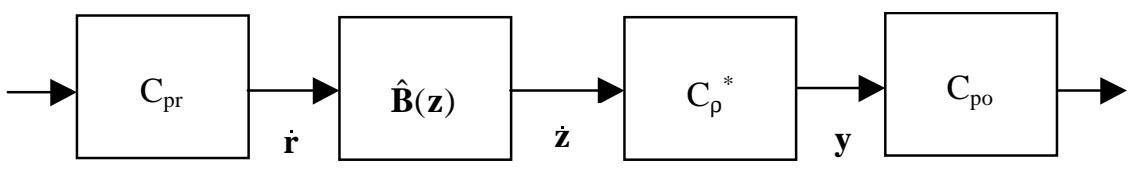

Figure 11

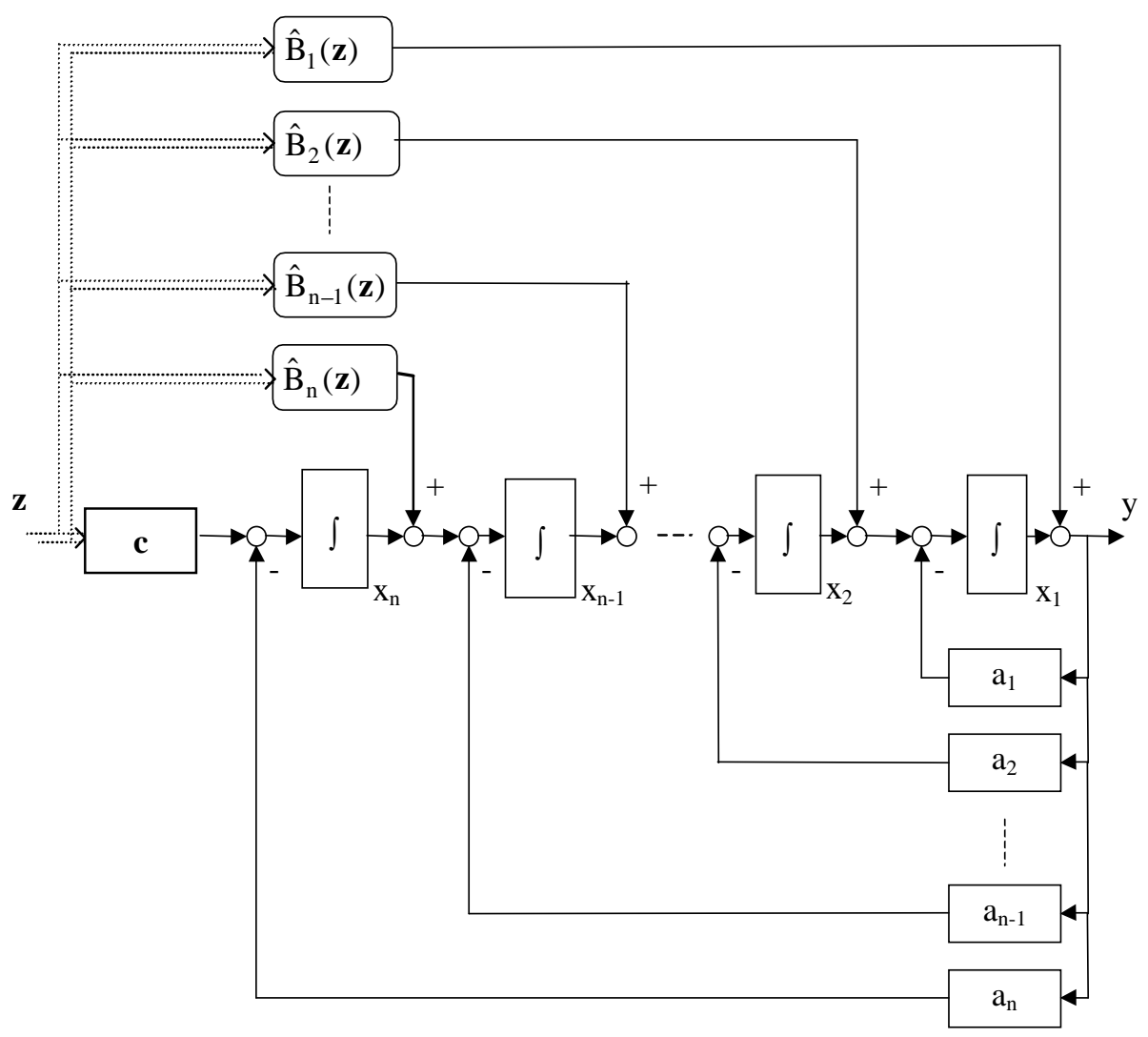


Figure 12

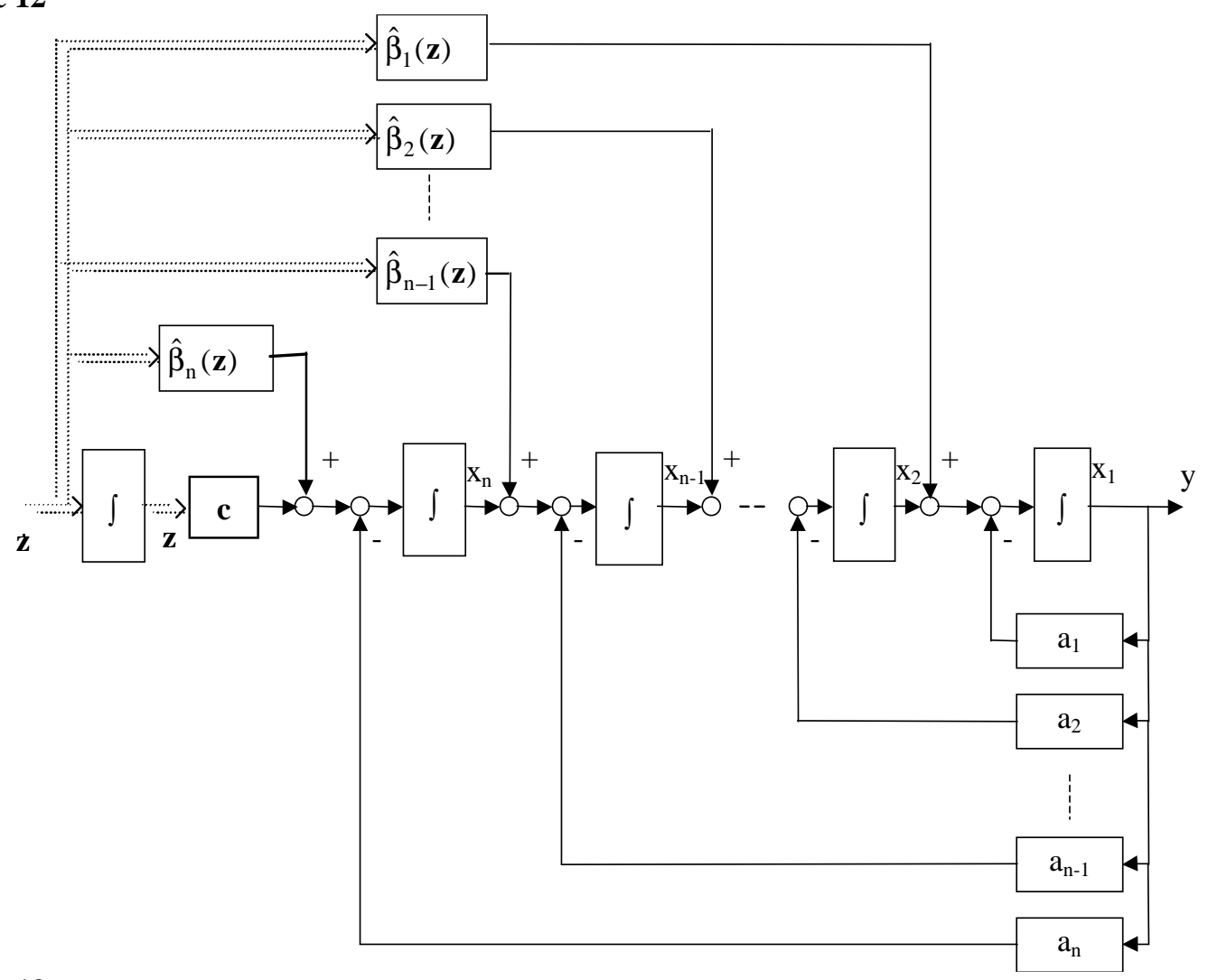

Figure 13

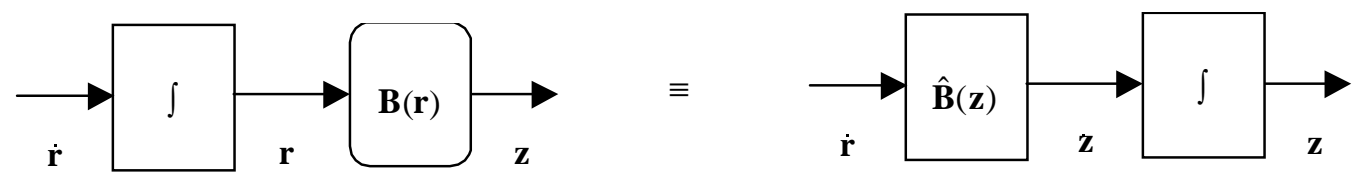

Figure 14

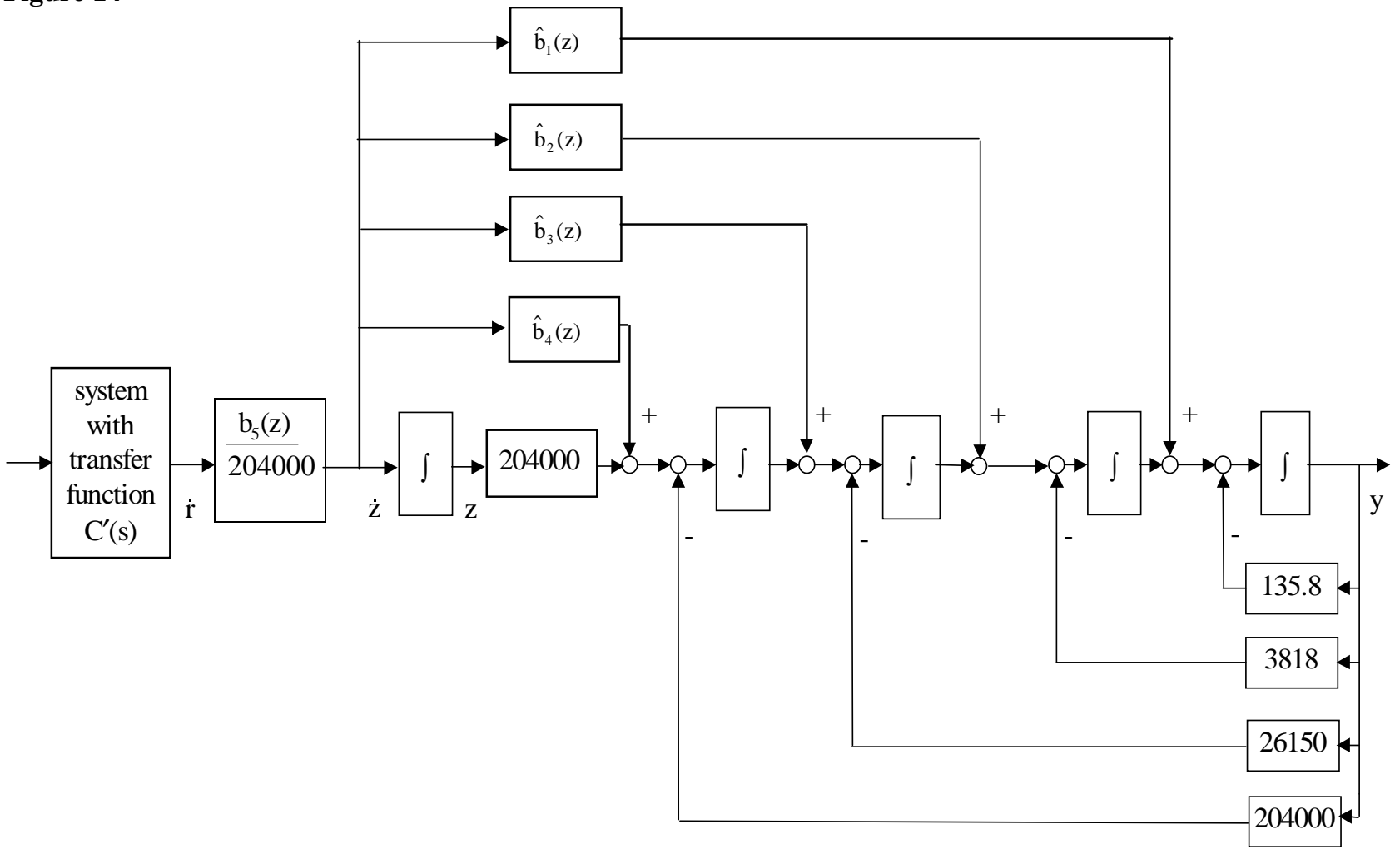

